\title{
Resonance neutron-capture cross sections of stable magnesium isotopes and their astrophysical implications
}

C. Massimi, ${ }^{1,2,{ }^{*}}$ P. Koehler, ${ }^{3}$ S. Bisterzo,${ }^{4}$ N. Colonna,${ }^{5}$ R. Gallino, ${ }^{4}$ F. Gunsing, ${ }^{6}$ F. Käppeler, ${ }^{7}$ G. Lorusso, ${ }^{5}$ A. Mengoni, ${ }^{8,9}$ M. Pignatari, ${ }^{10}$ G. Vannini, ${ }^{1,2}$ U. Abbondanno, ${ }^{11}$ G. Aerts, ${ }^{6}$ H. Álvarez,${ }^{12}$ F. Álvarez-Velarde, ${ }^{13}$ S. Andriamonje, ${ }^{6}$ J. Andrzejewski, ${ }^{14}$ P. Assimakopoulos, ${ }^{15, \dagger}$ L. Audouin, ${ }^{16}$ G. Badurek, ${ }^{17}$ M. Barbagallo, ${ }^{5}$ P. Baumann, ${ }^{18}$ F. Bečváŕ, ${ }^{19}$ F. Belloni,${ }^{11}$ M. Bennett, ${ }^{20}$ E. Berthoumieux, ${ }^{6}$ M. Calviani,${ }^{9}$ F. Calviño,${ }^{21}$ D. Cano-Ott,${ }^{13}$ R. Capote,${ }^{8,22}$ C. Carrapiço,,${ }^{23,6}$ A. Carrillo de Albornoz, ${ }^{23}$ P. Cennini, ${ }^{9}$ V. Chepel, ${ }^{24}$ E. Chiaveri, ${ }^{9}$ G. Cortes,${ }^{25}$ A. Couture, ${ }^{26}$ J. Cox,${ }^{26}$ M. Dahlfors, ${ }^{9}$ S. David,${ }^{16}$ I. Dillmann, ${ }^{7}$ R. Dolfini, ${ }^{27}$ C. Domingo-Pardo, ${ }^{28}$ W. Dridi, ${ }^{6}$ I. Duran, ${ }^{12}$ C. Eleftheriadis,${ }^{29}$ M. Embid-Segura, ${ }^{13}$

L. Ferrant,${ }^{16, \dagger}$ A. Ferrari, ${ }^{9}$ R. Ferreira-Marques, ${ }^{24}$ L. Fitzpatrick,${ }^{9}$ H. Frais-Koelbl,${ }^{8}$ K. Fujii, ${ }^{11}$ W. Furman,${ }^{30}$ I. Goncalves, ${ }^{23}$ E. González-Romero, ${ }^{13}$ A. Goverdovski, ${ }^{31}$ F. Gramegna, ${ }^{32}$ E. Griesmayer, ${ }^{8}$ C. Guerrero, ${ }^{13}$ B. Haas, ${ }^{33}$ R. Haight, ${ }^{34}$ M. Heil,${ }^{35}$ A. Herrera-Martinez, ${ }^{9}$ F. Herwig, ${ }^{36}$ R. Hirschi, ${ }^{20}$ M. Igashira,${ }^{37}$ S. Isaev, ${ }^{16}$ E. Jericha, ${ }^{17}$ Y. Kadi, ${ }^{9}$ D. Karadimos, ${ }^{15}$ D. Karamanis,,${ }^{15}$ M. Kerveno, ${ }^{18}$ V. Ketlerov, ${ }^{30}$ V. Konovalov ${ }^{29}$ S. Kopecky, ${ }^{38}$ E. Kossionides,${ }^{39}$ M. Krtička, ${ }^{19}$ C. Lampoudis, ${ }^{29,6}$ H. Leeb, ${ }^{17}$ C. Lederer ${ }^{40}$ A. Lindote, ${ }^{24}$ I. Lopes,${ }^{24}$ R. Losito, ${ }^{9}$ M. Lozano, ${ }^{22}$ S. Lukic, ${ }^{18}$

J. Marganiec, ${ }^{14}$ L. Marques, ${ }^{23}$ S. Marrone,${ }^{5}$ T. Martínez, ${ }^{13}$ P. Mastinu, ${ }^{32}$ E. Mendoza, ${ }^{13}$ P. M. Milazzo, ${ }^{11}$ C. Moreau, ${ }^{11}$ M. Mosconi, ${ }^{7}$ F. Neves,${ }^{24}$ H. Oberhummer, ${ }^{17}$ S. O'Brien, ${ }^{26}$ M. Oshima, ${ }^{41}$ J. Pancin, ${ }^{6}$ C. Papachristodoulou, ${ }^{15}$ C. Papadopoulos, ${ }^{42}$ C. Paradela, ${ }^{12}$ N. Patronis, ${ }^{15}$ A. Pavlik,${ }^{40}$ P. Pavlopoulos,,${ }^{43}$ L. Perrot, ${ }^{6}$ M. T. Pigni, ${ }^{17}$ R. Plag, ${ }^{7}$ A. Plompen, ${ }^{38}$ A. Plukis, ${ }^{6}$ A. Poch ${ }^{25}$ J. Praena, ${ }^{22}$ C. Pretel, ${ }^{25}$ J. Quesada, ${ }^{22}$ T. Rauscher, ${ }^{10}$ R. Reifarth, ${ }^{34}$ G. Rockefeller, ${ }^{34}$ M. Rosetti, ${ }^{44}$ C. Rubbia, ${ }^{27}$ G. Rudolf, ${ }^{18}$ J. Salgado, ${ }^{23}$ C. Santos, ${ }^{23}$ L. Sarchiapone, ${ }^{9}$ R. Sarmento, ${ }^{23}$ I. Savvidis,${ }^{29}$ C. Stephan, ${ }^{16}$ G. Tagliente, ${ }^{5}$ J. L. Tain, ${ }^{28}$ D. Tarrío, ${ }^{12}$ L. Tassan-Got, ${ }^{16}$ L. Tavora,${ }^{23}$ R. Terlizzi, ${ }^{5}$ P. Vaz, ${ }^{23}$ A. Ventura, ${ }^{44}$ D. Villamarin, ${ }^{13}$ V. Vlachoudis, ${ }^{9}$

R. Vlastou, ${ }^{42}$ F. Voss,${ }^{7}$ S. Walter, ${ }^{7}$ H. Wendler, ${ }^{9}$ M. Wiescher, ${ }^{26}$ and K. Wisshak ${ }^{7}$

(n_TOF Collaboration)

${ }^{1}$ Dipartimento di Fisica, Università di Bologna, Bologna, Italy

${ }^{2}$ Istituto Nazionale di Fisica Nucleare, Bologna, Italy

${ }^{3}$ Oak Ridge National Laboratory, Physics Division, Oak Ridge, Tennessee 37831-6369, USA

${ }^{4}$ Dipartimento di Fisica Generale, Università di Torino, Torino, Italy

${ }^{5}$ Istituto Nazionale di Fisica Nucleare, Bari, Italy

${ }^{6} \mathrm{CEA} /$ Saclay, IRFU, Gif-sur-Yvette, France

${ }^{7}$ Karlsruhe Institute of Technology (KIT), Campus Nord, Institut für Kernphysik, Germany

${ }^{8}$ International Atomic Energy Agency (IAEA), Nuclear Data Section, Vienna, Austria

${ }^{9}$ CERN, Geneva, Switzerland

${ }^{10}$ Department of Physics, University of Basel, Switzerland

${ }^{11}$ Istituto Nazionale di Fisica Nucleare, Trieste, Italy

${ }^{12}$ Universidade de Santiago de Compostela, Santiago de Compostela, Spain

${ }^{13}$ Centro de Investigaciones Energeticas Medioambientales y Tecnologicas, Madrid, Spain

${ }^{14}$ University of $\mathrm{Lodz}, \mathrm{Lodz}$, Poland

${ }^{15}$ University of Ioannina, Ioannina, Greece

${ }^{16}$ Centre National de la Recherche Scientifique/IN2P3-IPN, Orsay, France

${ }^{17}$ Atominstitut der Österreichischen Universitäten, Technische Universität Wien, Vienna, Austria

${ }^{18}$ Centre National de la Recherche Scientifique/IN2P3-IReS, Strasbourg, France

${ }^{19}$ Charles University, Prague, Czech Republic

${ }^{20}$ Keele University, Newcastle-under-Lyme, Staffordshire, United Kingdom

${ }^{21}$ Universidad Politecnica de Madrid, Madrid, Spain

${ }^{22}$ Universidad de Sevilla, Seville, Spain

${ }^{23}$ Instituto Tecnológico e Nuclear (ITN), Lisbon, Portugal

${ }^{24}$ LIP-Coimbra and Departamento de Fisica da Universidade de Coimbra, Coimbra, Portugal

${ }^{25}$ Universitat Politecnica de Catalunya, Barcelona, Spain

${ }^{26}$ University of Notre Dame, Notre Dame, USA

${ }^{27}$ Università degli Studi Pavia, Pavia, Italy

${ }^{28}$ Instituto de Física Corpuscular, CSIC-Universidad de Valencia, Valencia, Spain

${ }^{29}$ Aristotle University of Thessaloniki, Thessaloniki, Greece

${ }^{30}$ Joint Institute for Nuclear Research, Frank Laboratory of Neutron Physics, Dubna, Russia

${ }^{31}$ Institute of Physics and Power Engineering, Kaluga region, Obninsk, Russia

${ }^{32}$ Istituto Nazionale di Fisica Nucleare, Laboratori Nazionali di Legnaro, Padua, Italy

${ }^{33}$ Centre National de la Recherche Scientifique/IN2P3-CENBG, Bordeaux, France

${ }^{34}$ Los Alamos National Laboratory, New Mexico, Los Alamos 87545, USA

${ }^{35}$ GSI Helmholtzzentrum für Schwerionenforschung GmbH, Darmstadt, Germany

${ }^{36}$ Department of Physics and Astronomy, University of Victoria, Victoria, British Columbia, Canada 


\author{
${ }^{37}$ Tokyo Institute of Technology, Tokyo, Japan \\ ${ }^{38}$ EC-JRC-IRMM, Geel, Belgium \\ ${ }^{39}$ NCSR, Athens, Greece \\ ${ }^{40}$ University of Vienna, Faculty of Physics, Vienna, Austria \\ ${ }^{41}$ Japan Atomic Energy Research Institute, Tokai-mura, Japan \\ ${ }^{42}$ National Technical University of Athens, Athens, Greece \\ ${ }^{43}$ Pôle Universitaire Léonard de Vinci, Paris La Défense, France \\ ${ }^{44}$ ENEA, Bologna, Italy
}

(Received 9 January 2012; revised manuscript received 22 February 2012; published 20 April 2012)

\begin{abstract}
We have measured the neutron capture cross sections of the stable magnesium isotopes ${ }^{24,25,26} \mathrm{Mg}$ in the energy range of interest to the $s$ process using the neutron time-of-flight facility $\mathrm{n} \_$TOF at CERN. Capture events from a natural metal sample and from samples enriched in ${ }^{25} \mathrm{Mg}$ and ${ }^{26} \mathrm{Mg}$ were recorded using the total energy method based on $\mathrm{C}_{6}{ }^{2} \mathrm{H}_{6}$ detectors. Neutron resonance parameters were extracted by a simultaneous resonance shape analysis of the present capture data and existing transmission data on a natural isotopic sample. Maxwellian-averaged capture cross sections for the three isotopes were calculated up to thermal energies of $100 \mathrm{keV}$ and their impact on $s$-process analyses was investigated. At $30 \mathrm{keV}$ the new values of the stellar cross section for ${ }^{24} \mathrm{Mg},{ }^{25} \mathrm{Mg}$, and ${ }^{26} \mathrm{Mg}$ are $3.8 \pm 0.2 \mathrm{mb}, 4.1 \pm 0.6 \mathrm{mb}$, and $0.14 \pm 0.01 \mathrm{mb}$, respectively.
\end{abstract}

DOI: 10.1103/PhysRevC.85.044615

PACS number(s): $26.20 . \mathrm{Kn}, 28.20 . \mathrm{Np}, 29.30 . \mathrm{Hs}, 21.10 . \mathrm{Hw}$

\section{INTRODUCTION}

The slow neutron-capture process ( $s$ process) $[1-4]$ in stars is responsible for the origin of about one half of the elemental abundances beyond iron that we observe today. In this process most of the neutrons are provided by the ${ }^{13} \mathrm{C}(\alpha, n){ }^{16} \mathrm{O}$ reaction and by the ${ }^{22} \mathrm{Ne}(\alpha, n)^{25} \mathrm{Mg}$ reaction. Most of the produced neutrons are captured by light species in competition with ${ }^{56} \mathrm{Fe}$, that is the main seed for $s$-process nucleosynthesis on heavy elements. Among light neutron poisons, ${ }^{25} \mathrm{Mg}$ and ${ }^{26} \mathrm{Mg}$ may have a relevant impact on neutron balance, and their neutroncapture cross sections need to be known with high precision, in order to obtain robust $s$-process calculations. Additionally, these results yield some constraints for the yet poorly known ${ }^{22} \mathrm{Ne}(\alpha, n){ }^{25} \mathrm{Mg}$ cross section by studying the states of the ${ }^{25} \mathrm{Mg}+n$ compound nucleus.

Another aspect of the capture cross section of the stable magnesium isotopes is related to the open question of the production of the radioisotope ${ }^{26} \mathrm{Al}$ in the cosmos. The sensitivity study of Iliadis et al. [5] has demonstrated that the cross section of the ${ }^{24} \operatorname{Mg}(n, \gamma)$ reaction is important for the origin of ${ }^{26} \mathrm{Al}$, because its main production mechanism in massive stars is strongly affected by the uncertainties of several cross sections, including that of ${ }^{24} \operatorname{Mg}(n, \gamma)$.

The capture cross sections of the $\mathrm{Mg}$ isotopes in current nuclear data libraries exhibit deficiencies in the resolved resonance region (RRR), in particular concerning the assignment of resonance spins. The respective evaluations are based on the Japanese Evaluated Nuclear Data Library (JENDL) 3.2 [6] version, which adopted the Brookhaven National Laboratory compilation [7] in the RRR. Because the energy range of interest for $s$-process temperatures is slightly larger than the one covered by the evaluation, Koehler [8] recently reanalyzed

*Cristian.Massimi@bo.infn.it

${ }^{\dagger}$ Deceased. existing data [9] to derive an improved set of resonance parameters. His analysis included very high resolution data for the total cross section obtained with a metallic sample of natural $\mathrm{Mg}$ and high-resolution capture data measured with an enriched ${ }^{25} \mathrm{Mg}$ sample, both from experiments at the Oak Ridge Electron Linear Accelerator (ORELA) neutron time-of-flight facility [10].

Although the evaluation of Ref. [8] is more accurate than that in Ref. [6], there are three problems [11] with the former work (Ref. [8]). First, the scattering widths given in Tables I and II of Ref. [8] are actually $\Gamma_{n}$ and not $g \Gamma_{n}$, where $g$ is the statistical spin factor. Second, and more importantly, the sample thickness used in the transmission analysis was $10 \%$ too small. Third, the transmission data used in that analysis were too much averaged near the $475-\mathrm{keV}$ resonance, so the quoted parameters for that resonance are not very accurate.

Previous experiments comprise a series of neutron timeof-flight (TOF) measurements on ${ }^{\text {nat }} \mathrm{Mg}$ as well as on enriched samples. Typically, these experiments covered only a limited energy region, and capture data were taken with large detectors, less suited for capture cross-section studies on isotopes in the mass region of $\mathrm{Mg}$, where the cross sections are by far dominated by the elastic-scattering channel. While the most abundant isotope ${ }^{24} \mathrm{Mg}$ has been investigated several times [12-15], there are few neutron data for ${ }^{25,26} \mathrm{Mg}$. Because the resonance-dominated capture cross sections of all three $\mathrm{Mg}$ isotopes are relatively small, the measurements can be strongly affected by various kinds of background and exhibit, therefore, rather large discrepancies. For instance, spin and parity of the first neutron resonance in ${ }^{25} \mathrm{Mg}+n$ have been reported as $J^{\pi}=3^{+}$[16], $3^{-}$[17], and $2^{+}$[18] but was assigned as $2^{-}$ in the JENDL evaluation, although the parity assignment of Ref. [18] is consistent with an independent ${ }^{26} \mathrm{Mg}(\gamma, n)^{25} \mathrm{Mg}$ experiment [19].

Concerning ${ }^{26} \mathrm{Mg}+n$ reaction, the observed differences between the only TOF data from Ref. [9] and activation experiments by Mohr et al. [20,21] are consistent with the 
TABLE I. Characteristics of the Mg samples.

\begin{tabular}{|c|c|c|c|c|c|}
\hline \multirow{2}{*}{$\begin{array}{l}\text { Sample } \\
\text { ID }\end{array}$} & \multicolumn{3}{|c|}{ Isotopic abundance (\%) } & \multirow{2}{*}{$\begin{array}{c}\text { Total } \\
\text { mass (g) }\end{array}$} & \multirow{2}{*}{ 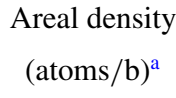 } \\
\hline & ${ }^{24} \mathrm{Mg}$ & ${ }^{25} \mathrm{Mg}$ & ${ }^{26} \mathrm{Mg}$ & & \\
\hline${ }^{\text {nat }} \mathrm{Mg}$ & 78.7 & 10.13 & 11.17 & 5.2393 & 0.03415 \\
\hline${ }^{25} \mathrm{Mg}$ & 3.05 & 95.75 & 1.20 & 3.1924 & 0.01234 \\
\hline${ }^{26} \mathrm{Mg}$ & 2.46 & 1.28 & 96.26 & 3.2301 & 0.01219 \\
\hline
\end{tabular}

${ }^{\mathrm{a}}$ Areal density of the $\mathrm{Mg}$ isotopes.

direct radiative capture (DRC) component [22] that is not covered by the TOF technique. For this part, the thermal capture cross section reported in Ref. [23] provides an additional constraint for normalization of the $1 / v$ slope of the cross section ( $v$ being the neutron velocity).

In view of the uncertain and incomplete cross-section data of the $\mathrm{Mg}$ isotopes, which may be due to the neutron sensitivity of previous experiments, a set of capture measurements was performed at $n \_$TOF to update the $(n, \gamma)$ cross sections of ${ }^{24,25,26} \mathrm{Mg}$. In these measurements particular care was devoted to minimizing systematic uncertainties due to scattered neutrons. In addition, enriched samples were used to improve the assignment of some doubtful resonances.

The experiment and the procedure for the data reduction are described in Secs. II and III, respectively. The resonance analysis is discussed in Sec. IV and the corresponding stellar cross sections are given in Sec. V. The astrophysical implications are illustrated in Sec. VI and the conclusions are in Sec. VII.

\section{EXPERIMENT}

The capture experiment was performed at $n_{-}$TOF, the neutron time-of-flight facility at CERN [24], which provides a white neutron spectrum from thermal to about $1-\mathrm{GeV}$ neutron energy. Neutrons are produced in a massive lead target by a pulsed $20-\mathrm{GeV}$ proton beam from the CERN/PS accelerator complex. This spallation neutron source is characterized by the high intensity of $7 \times 10^{12}$ protons per pulse, a short pulse width of $6 \mathrm{~ns}$, a low repetition rate of $0.4 \mathrm{~Hz}$, and a long flight path of $185 \mathrm{~m}$. Two collimators are present in the neutron beam. They provide a nearly symmetric Gaussian-shaped beam profile at the sample position, with an energy-dependent standard deviation, which is about $0.77 \mathrm{~cm}$ at low neutron energies. A full description of its characteristics and performance can be found in Refs. $[25,26]$. The background level is kept low, in the experimental area, thanks to several massive concrete and iron shieldings and by means of a strong sweeping magnet.

\section{A. Capture apparatus}

The capture apparatus consisted of two $\mathrm{C}_{6}{ }^{2} \mathrm{H}_{6}$ liquid scintillators. The deuterated benzene liquid scintillators used in the present measurement consisted of cylindrical cells $127.3 \mathrm{~mm}$ in diameter and $78 \mathrm{~mm}$ in length with an active volume of about $1000 \mathrm{~cm}^{3}$. Deuterated benzene was chosen for its very small neutron sensitivity. The neutron sensitivity of

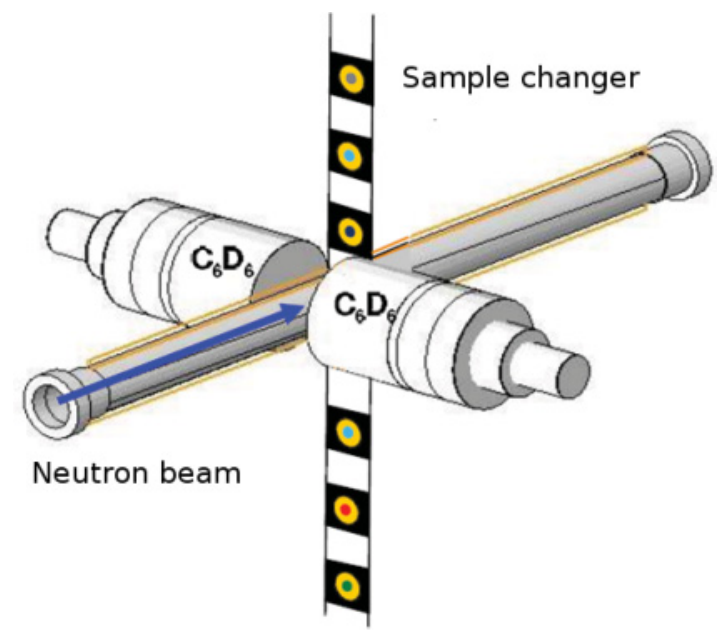

FIG. 1. (Color online) Sketch of sample changer and detectors in the experimental area at a flight path of $185 \mathrm{~m}$ (D denotes deuterium, $\left.{ }^{2} \mathrm{H}\right)$.

the detectors was further minimized by coupling a thin carbonfiber cell directly to the EMI 9823QKA photomultipliers [27]. The detectors were placed perpendicular to the beam, $9.2 \mathrm{~cm}$ upstream from the sample center in order to reduce the background due to scattered photons. This geometrical configuration also allowed us to reduce the effects of the angular distribution from primary neutron capture $\gamma$ rays following neutron capture in $\ell=1 p$-wave resonances. The setup of the sample-detector geometry is sketched in Fig. 1. The total energy detection method in combination with the pulse height weighting technique (PHWT) [28] was used for this experiment. The neutron fluence at the sample position, about $185 \mathrm{~m}$ from the neutron source, was measured with a well-calibrated ${ }^{6} \mathrm{Li}$-based neutron monitor [29]. It is an in-beam detector, consisting of a ${ }^{6} \mathrm{Li}$ deposit $\left(300 \mathrm{mg} / \mathrm{cm}^{2}\right.$ and $6 \mathrm{~cm}$ in diameter) on a Mylar foil and four off-beam silicon $\left(6 \times 4 \mathrm{~cm}^{2}\right)$ detectors measuring the particles from the ${ }^{6} \mathrm{Li}(n$, $\alpha)^{3} \mathrm{H}$ reaction. The monitor was located about $3 \mathrm{~m}$ upstream of the sample position.

The detector signals were recorded using fast digitizers with a sampling rate of 500 Msamples/s [30]. This configuration made it possible to record the detector signals over the entire TOF interval from relativistic neutron energies down to approximately $1 \mathrm{eV}$. The effective length of the flight path, $L=185.07 \pm 0.01 \mathrm{~m}$, was calibrated using the first $s$-wave resonances of $\mathrm{Au}$ as explained in Ref. [31]. The TOF data were converted to neutron energy by

$$
E_{n}=m_{n} c^{2}\left[\frac{c}{\sqrt{c^{2}-\left(\frac{L}{\Delta t}\right)^{2}}}-1\right]
$$

where $m_{n}$ is the neutron mass and $c$ the speed of light. The TOF interval of a neutron $\Delta t$ was determined by the time between the start signal, based on the reference signal provided by the prompt $\gamma$-flash $t_{\gamma}$, and the stop signal $t_{n}$ (both 
detected in the $\mathrm{C}_{6}{ }^{2} \mathrm{H}_{6}$ detectors) according to the following: $\Delta t=t_{n}-t_{\gamma}+L / c$.

\section{B. Samples and measurements}

Enriched samples of ${ }^{25} \mathrm{Mg}$ and ${ }^{26} \mathrm{Mg}$ were borrowed from the Science-Technical Centre "Stable Isotopes" (Obninsk, Russia) in the form of magnesium oxide powder. The powder was sealed in very thin aluminum cans with total masses of $350 \mathrm{mg}$. The enriched samples were complemented by a metal disk of natural magnesium. All samples were $22 \mathrm{~mm}$ in diameter.

The composition of the samples is listed in Table I. The specified impurities of the enriched samples included traces of $\mathrm{Be}, \mathrm{Sb}, \mathrm{Fe}, \mathrm{Al}, \mathrm{Sn}, \mathrm{Mn}, \mathrm{Cu}, \mathrm{Ca}, \mathrm{Mo}, \mathrm{Ni}, \mathrm{Ag}$, and $\mathrm{Pb}$ [32]. From the resonance shape analysis (RSA) of the capture data, some traces of In were found in addition. The concentration of impurities was verified and it resulted to be very low. For instance, the most important impurities $\left({ }^{115} \mathrm{In},{ }^{121,123} \mathrm{Sb},{ }^{117} \mathrm{Sn}\right.$, and ${ }^{95} \mathrm{Mo}$ ) were at the level of tens of ppm. The mass of the samples was quoted in the accompanying documentation without uncertainties. Furthermore, no information was available on the procedure used for the preparation, nor on the final homogeneity of the powder samples. Because $\mathrm{MgO}$ is highly hygroscopic, the $\mathrm{Mg}$ content might have been overestimated by the absorption of moisture before the powder was sealed in the Al cans. Unfortunately, it was not possible to heat the samples for removing absorbed water as described in Ref. [20].

In fact, the comparative analysis of the first $s$-wave resonance at $19.86 \mathrm{keV}$ in the ${ }^{25} \mathrm{Mg}(n, \gamma)$ cross section, which was observed with the enriched ${ }^{25} \mathrm{MgO}$ sample and with the metallic natural sample, provided clear evidence that the quoted ${ }^{25} \mathrm{Mg}$ mass was overestimated by about $30 \%$ (see Sec. IV B). An alternative explanation could be possible inhomogeneities of the sample, related to the spatial distribution of the powder inside the canning. Nevertheless, a procedure described in the analysis section allowed determination of the ${ }^{25} \mathrm{Mg}$ mass with an uncertainty of approximately $12 \%$. Unfortunately, the same procedure could not be applied to the ${ }^{26} \mathrm{Mg}$ sample. Therefore, the cross section presented herein may be underestimated by as much as $30 \%$ for this isotope.

Additional samples of $\mathrm{Au}, \mathrm{Pb}$, and $\mathrm{C}$ (all $22 \mathrm{~mm}$ in diameter) have been used in the experiment. The $\mathrm{Pb}$ and $\mathrm{C}$ (corresponding to an areal density of $2.99 \times 10^{-3}$ atoms $/ \mathrm{b}$ and $2.018 \times 10^{-2}$ atoms $/ \mathrm{b}$, respectively) disks were used to determine various background components. A gold sample, $0.25 \mathrm{~mm}$ in thickness (corresponding to an areal density of $1.498 \times 10^{-3}$ atoms $/ \mathrm{b}$ ), served to normalize the capture data via the saturated resonance technique [33]. This technique can be applied when the macroscopic total cross section is much larger than unity. In this particular case, all incoming neutrons, with energies in the vicinity of the resonance energy, interact with the sample. Therefore, since all neutrons are absorbed in the sample, the probability of a capture event in the sample is 1 .

The measurements with the different samples were cycled every 2 days and they were interspersed with energy calibration of the scintillators.

\section{DATA REDUCTION}

The use of the PHWT, by which the TOF spectrum is modified on the basis of the signal amplitude, requires a careful energy calibration of the capture detectors in combination with proper study of the detector resolution [34]. During the entire experiment, the pulse height response of the $\mathrm{C}_{6}{ }^{2} \mathrm{H}_{6}$ detectors was calibrated in regular intervals with standard sources, i.e., with ${ }^{137} \mathrm{Cs}$, ${ }^{60} \mathrm{Co}$, and a composite ${ }^{238} \mathrm{Pu} / \mathrm{C}$ source, which yields $6.13-\mathrm{MeV} \gamma$ rays through the ${ }^{13} \mathrm{C}(\alpha, n){ }^{16} \mathrm{O}^{*}$ reaction. Data were taken with a digitizer threshold corresponding to a deposited energy of about $160 \mathrm{keV}$, but a fixed threshold of $200 \mathrm{keV}$ was later applied in the off-line processing.

By the use of fast digitizers for data acquisition the dead time could be reduced to an effective value of less than $25 \mathrm{~ns}$, related to the pulse reconstruction algorithm. In the off-line event processing, we applied a fixed dead time of $30 \mathrm{~ns}$ and used this value in the calculation of the correction due to counting-rate losses. When an event was observed, all subsequent signals occurring within the dead time of $30 \mathrm{~ns}$ in both detectors were discarded in order to eliminate coincidence counting. The dead time correction never exceeded $1 \%$.

\section{A. Capture yield}

The capture yield $Y\left(E_{n}\right)$, which represents the probability of a neutron to be captured by the sample, can be deduced from the background-subtracted counts in the TOF spectrum $C\left(E_{n}\right)$ registered by the $\mathrm{C}_{6}{ }^{2} \mathrm{H}_{6}$ array,

$$
C\left(E_{n}\right)=Y\left(E_{n}\right) \Phi\left(E_{n}\right) A \varepsilon_{c},
$$

where $\Phi\left(E_{n}\right)$ represents the intensity of the neutron beam, $A$ the sample area, and $\varepsilon_{c}$ the efficiency for detecting a capture event.

In the PHWT, the proportionality of the capture efficiency to the total $\gamma$ energy released in the capture event $\varepsilon_{c} \propto$ $E_{c}$, where $E_{c}$ is the sum of the neutron separation energy and the kinetic energy $\left(E_{c}=S_{n}+K_{\text {c.m. }}\right)$, is obtained by a weighting function, which modifies the detection efficiency so $\varepsilon_{c}$ becomes independent of the $\gamma$ cascade. The weighted count rate spectrum is

$$
C_{W}\left(E_{n}\right)=N Y\left(E_{n}\right) \Phi\left(E_{n}\right) E_{c},
$$

where the absolute normalization $N$ of the capture data is obtained by means of the saturated resonance in $\mathrm{Au}$ at $4.9 \mathrm{eV}$. Since the Au disk was $0.25 \mathrm{~mm}$ in thickness, the attenuation of the $\gamma$ rays in the Au sample was considered as described in Ref. [28]. The corresponding correction of the normalization constant was of the order of $1 \%$.

The measured capture yields are shown in Fig. 2.

\section{B. Background studies}

The background components have been determined by comparison of the $\mathrm{Mg}$ capture yields with the respective yields measured with the $\mathrm{Pb}$ and $\mathrm{C}$ samples as illustrated in Fig. 3.

The main source of background in the $\mathrm{keV}$ region is generated by in-beam $\gamma$ rays, which are scattered from the 


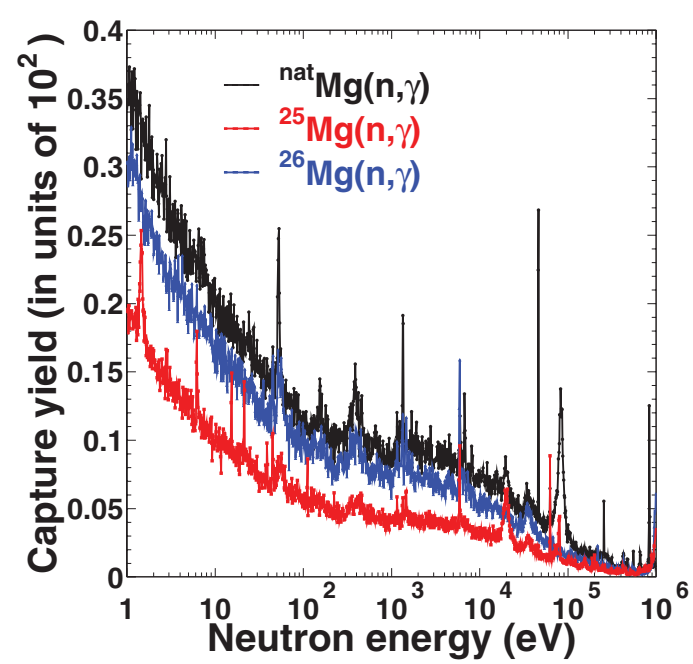

FIG. 2. (Color online) Capture yield of the ${ }^{\text {nat }} \operatorname{Mg}(n, \gamma),{ }^{25} \operatorname{Mg}(n$, $\gamma)$, and ${ }^{26} \operatorname{Mg}(n, \gamma)$ reactions.

sample and detected by the capture setup. These $\gamma$ rays arise mainly from neutron capture on hydrogen in the water moderator surrounding the spallation target and are responsible for most of the background in the $\mathrm{keV}$ region. This component is most pronounced in the yield of the $\mathrm{Pb}$ sample, which is particularly sensitive to in-beam $\gamma$ rays due to high atomic number of lead. Accordingly, the effect for the $\mathrm{C}$ and $\mathrm{Mg}$ samples is much weaker as illustrated in Fig. 3.

Another important background is produced by samplescattered neutrons, which are captured in the detection setup and in surrounding materials. This background component is studied with the $\mathrm{C}$ sample that can be considered as a pure neutron-scatterer sample. The shape of the carbon yield is very similar to that of ${ }^{25} \mathrm{Mg}$, because the nonresonant elastic cross section $\sigma_{n}$ (the so-called potential scattering) is smooth for both elements in this region and the elastic yield $n \sigma_{n}$ (where $n$ is the areal density in atoms per barn) is similar for both samples.

The comparison of the two components shows that the overall background was dominated by the effect of sample-

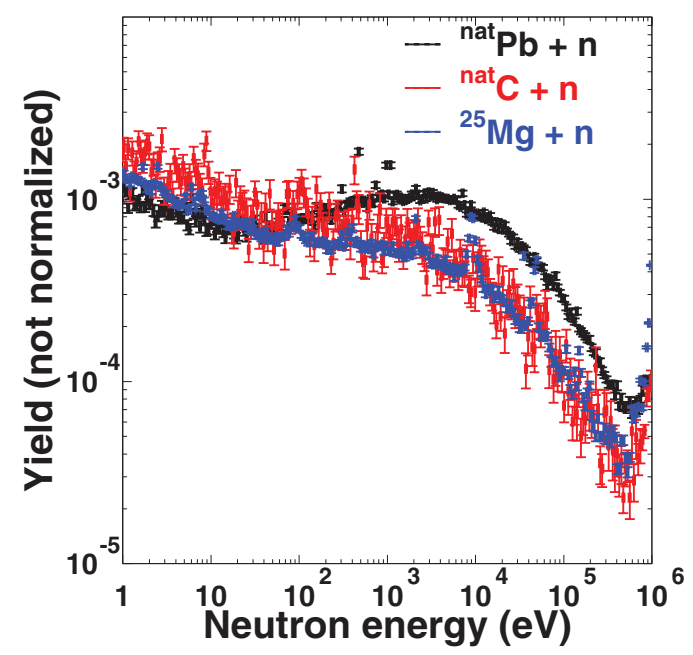

FIG. 3. (Color online) Capture yield of the ${ }^{25} \mathrm{Mg}+n$ reaction together with the spectra of the $\mathrm{C}$ and $\mathrm{Pb}$ measurements. scattered neutrons. Since the background displays a smooth, nonresonant behavior as a function of energy, it was not subtracted but was fitted in the resonance analysis. In this way, the uncertainty related to the background was included in the uncertainty of the resonance parameters.

\section{SIMULTANEOUS RESONANCE SHAPE ANALYSIS}

The present capture data were analyzed together with transmission data from ORELA [9], available from the Experimental Nuclear Reaction Data (EXFOR) database [35], using the $R$-matrix code SAMMY [36]. A simultaneous resonance shape analysis of capture and transmission data results in much more reliable resonance parameters than can be obtained through independent analyses of the various data sets. Experimental effects due to neutron multiple scattering in the sample, self-shielding (i.e., shielding of the inner atoms in the sample by the outer atoms closer to the surface), Doppler broadening, and experimental resolution are properly taken into account within the SAMMY code.

As shown in Eq. (3), the efficiency, and, hence, the calculated capture yield, is inversely proportional to $E_{c}$. However, only a single $S_{n}$ (typically chosen to be that of the most abundant isotope in the sample) can be used in weighting the data. Therefore, in the analysis of the capture data, the abundances of the other $\mathrm{Mg}$ isotopes in the sample must be scaled according to their $S_{n}$ value. In particular, the neutron separation energies used were 7.33, 11.09, and 6.44 MeV for ${ }^{25} \mathrm{Mg},{ }^{26} \mathrm{Mg}$, and ${ }^{27} \mathrm{Mg}$, respectively.

Resonance parameters reported in Ref. [8] were used as initial values in the RSA, with the following exceptions. An average reaction width $\Gamma_{\gamma}=3.5 \mathrm{eV}$ was kept fixed to fit capture data when a resonance was not visible in the transmission data. Spin-parity assignment of resonances above $500 \mathrm{keV}$ were taken from known results of a neutron elasticscattering experiment [13]. All resonances up to $700 \mathrm{keV}$ were included in the $R$ matrix. The availability of capture data in the full energy range allowed us to assign the observed resonances to the respective isotopes in the fit of the transmission data up to $700 \mathrm{keV}$. The RSA of the capture data was limited to below about $700-\mathrm{keV}$ neutron energy, where inelastic scattering starts to interfere.

In the fitting procedure, the resonance energy and the partial widths $\left(\Gamma_{n}\right.$ and $\left.\Gamma_{\gamma}\right)$ were allowed to vary while spin and angular momentum were kept fixed. The nuclear radii for ${ }^{24} \mathrm{Mg}+n$ and ${ }^{25} \mathrm{Mg}+n$ were allowed to vary in the fits of the transmission data as explained in Ref. [8]. The results are radii of 5.4 and $3.8 \mathrm{fm}$ for $s$ and $p$ waves in ${ }^{24} \mathrm{Mg}+n$, respectively, and $5.1 \mathrm{fm}$ common to $s$ and $p$ waves in ${ }^{25} \mathrm{Mg}+n$. Figure 4 shows the quality of the fit to the transmission data.

Parameters of the resonances at negative energy were changed with respect to the assumptions in Ref. [6] to reproduce the thermal-neutron capture cross section reported in Ref. [23]. Their energy was taken from the level scheme in Ref. [37].

\section{A. ${ }^{24} \mathrm{Mg}+\boldsymbol{n}$ resonances}

The capture yield considered for this analysis was obtained from the ${ }^{\text {nat }} \mathrm{Mg}+n$ measurement. The problems described in 


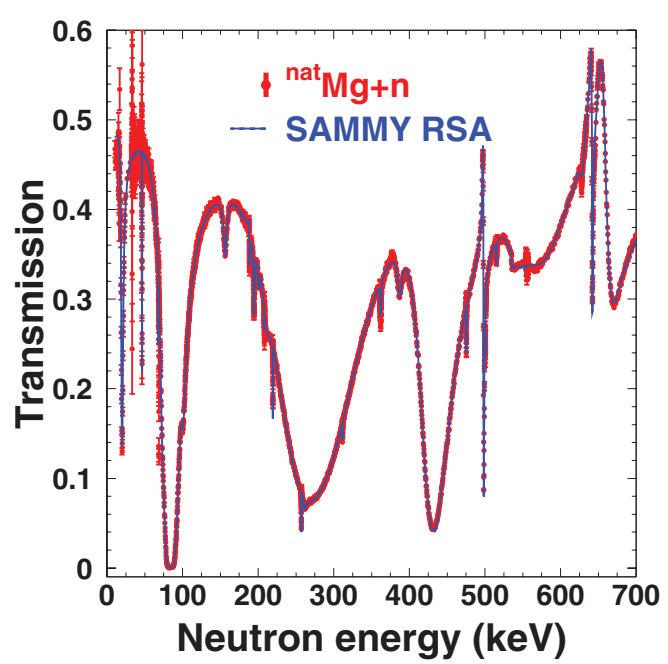

FIG. 4. (Color online) ${ }^{\text {nat }} \mathrm{Mg}+n$ transmission data (red symbols) and the SAMMY fit (blue line).

Sec. II B for the oxide samples do not apply for this metal sample.

In Table II the results of the RSA are reported together with the uncertainties from the fitting procedure. Examples of the fits are given in Figs. 4 and 5.

In the present analysis, the $68.5-\mathrm{keV}$ resonance, assigned to ${ }^{24} \mathrm{Mg}$ in the literature, has been found to be a ${ }^{26} \mathrm{Mg}$ resonance. Indeed, it was visible in the ${ }^{26} \mathrm{Mg}(n, \gamma)$ data and with much reduced size in the ${ }^{\text {nat }} \operatorname{Mg}(n, \gamma)$. Moreover, the doubtful resonance at $177 \mathrm{keV}$ was confirmed as belonging to ${ }^{24} \mathrm{Mg}$.

The capture kernels

$$
\omega \gamma=g \Gamma_{\gamma} \Gamma_{n} /\left(\Gamma_{\gamma}+\Gamma_{n}\right),
$$

calculated from present resonance parameters, are compared to those from Ref. [9] in Fig. 6. In particular ratios of the capture kernels as a function of neutron energy and of the $g \Gamma_{n} / \Gamma_{\gamma}$ values are shown. It has been shown [38] that some kernels

TABLE II. ${ }^{24} \mathrm{Mg}+n$ resonance parameters extracted from the simultaneous $R$-matrix analysis. The quoted uncertainties were obtained by the SAMMY fit. Spin and parity from Ref. [8] and Ref. [13].

\begin{tabular}{lcccc}
\hline \hline$E_{n}(\mathrm{keV})$ & $\ell$ & $J^{\pi}$ & $\Gamma_{\gamma}(\mathrm{eV})$ & $\Gamma_{n}(\mathrm{eV})$ \\
\hline-100 & 0 & $0.5^{+}$ & 13 & 3000 \\
$46.347 \pm 0.001$ & $(1)$ & $\left(0.5^{-}\right)$ & $1.4 \pm 0.2$ & $1.44 \pm 0.06$ \\
$83.940 \pm 0.004$ & 1 & $1.5^{-}$ & $4.1 \pm 0.2$ & $7607 \pm 4$ \\
$176.67 \pm 0.01$ & $(1)$ & $\left(0.5^{-}\right)$ & $3.5^{\mathrm{a}}$ & $0.4 \pm 0.2$ \\
$257.182 \pm 0.001$ & $(2)$ & $\left(1.5^{+}\right)$ & $1.8 \pm 0.1$ & $20.9 \pm 0.5$ \\
$267.48 \pm 0.01$ & 1 & $0.5^{-}$ & $7 \pm 3$ & $83270 \pm 20$ \\
$430.79 \pm 0.01$ & 1 & $1.5^{-}$ & $6.7 \pm 0.6$ & $28180 \pm 20$ \\
$475.359 \pm 0.004$ & 2 & $2.5^{+}$ & $1.2 \pm 0.2$ & $13.9 \pm 0.5$ \\
$498.285 \pm 0.004$ & 1 & $1.5^{-}$ & $0.25 \pm 0.1$ & $752 \pm 2$ \\
$551.04 \pm 0.03$ & $(2)$ & $\left(2.5^{+}\right)$ & $8 \pm 7$ & $1.2 \pm 0.4$ \\
$642.012 \pm 0.004$ & 1 & $0.5^{-}$ & $1.5 \pm 0.3$ & $1459 \pm 8$ \\
$659.95 \pm 0.01$ & 0 & $0.5^{+}$ & $(13 \pm 1)$ & $17470 \pm 40$ \\
\hline \hline
\end{tabular}

${ }^{a}$ Assumed reaction width. See text for details.
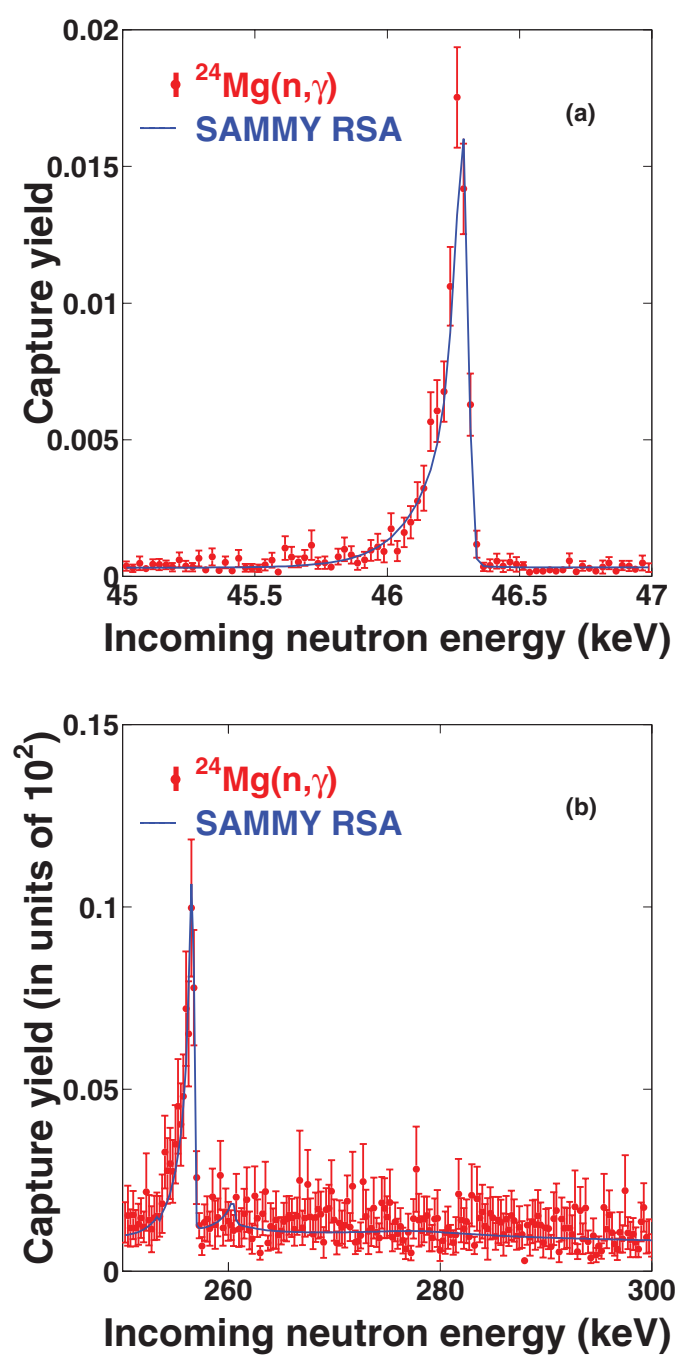

FIG. 5. (Color online) Fits of the capture yield of the ${ }^{24} \mathrm{Mg}+n$ reaction in different energy regions.

from previous ORELA measurements for resonances having large $g \Gamma_{n} / \Gamma_{\gamma}$ were systematically too large, presumably due to an underestimation of the neutron-scattering background (so-called neutron sensitivity effect). The differences between the present capture kernels and those of Ref. [9] do not appear to indicate this neutron sensitivity effect.

\section{B. ${ }^{25} \mathrm{Mg}+n$ resonances}

The capture yield of ${ }^{25} \mathrm{Mg}$ was obtained from the spectrum measured with the enriched oxide sample after its areal density was scaled to reproduce the resonance parameters determined from the natural $\mathrm{Mg}$ sample, where the first $s$-wave resonance in ${ }^{25} \mathrm{Mg}+n$ at $19.86 \mathrm{keV}$ is well isolated. Without that correction, $\Gamma_{\gamma}$ values of $1.7 \pm 0.2$ and $1.16 \pm 0.06 \mathrm{eV}$ were found using the data measured with the natural and the enriched $\mathrm{MgO}$ sample, respectively. This disagreement suggested that the areal density of the oxide sample was $27.5 \%$ too high, indicating a significant water contamination of the oxide sample. 

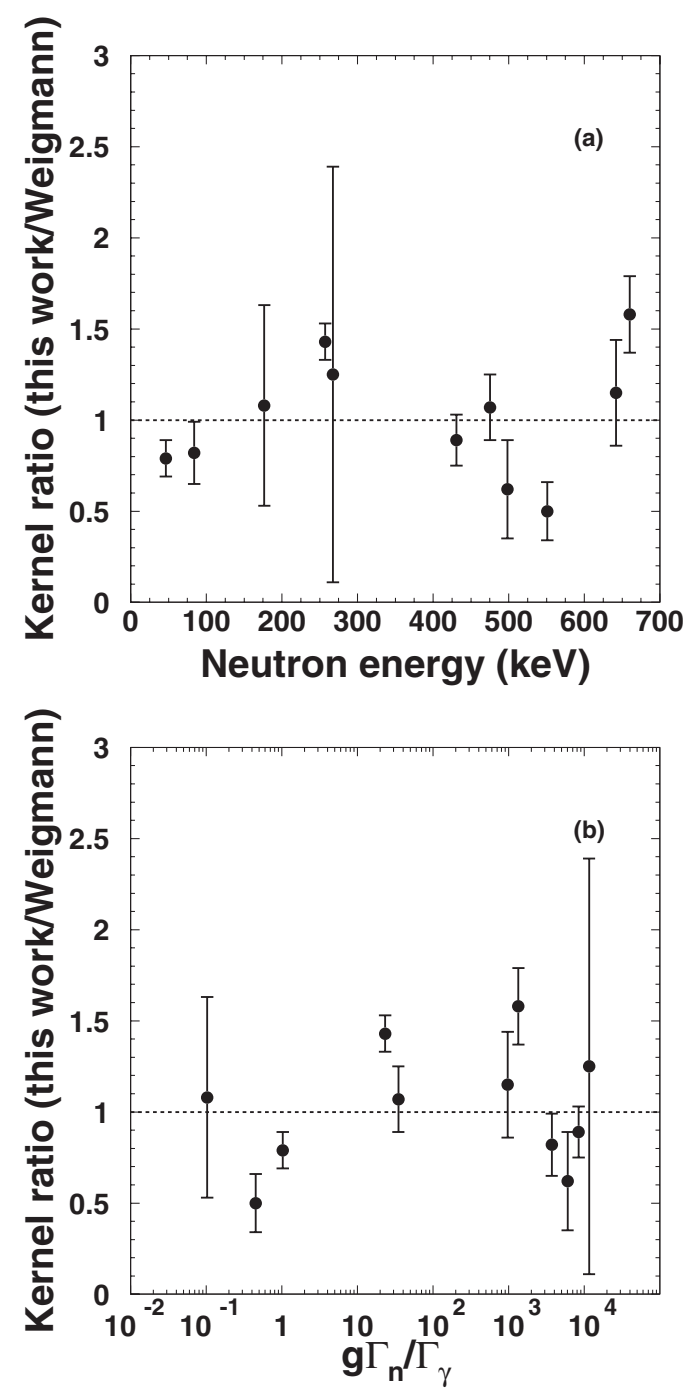

FIG. 6. Capture kernel ratios of the present work to Weigmann [9] as functions of neutron energy (a) and $g \Gamma_{n} / \Gamma_{\gamma}$ values (b) for ${ }^{24} \mathrm{Mg}$ resonances.

If the shape of a resonance is distorted by experimental effects, e.g., by Doppler and resolution broadening, the remaining observable is the capture kernel (proportional to the area under the resonance) and the areal density of the sample [39]. Accordingly, the RSA is sensitive to $n g \Gamma_{\gamma} \Gamma_{n} /\left(\Gamma_{\gamma}+\Gamma_{n}\right)$. Because $\Gamma_{n} \gg \Gamma_{\gamma}$ for the $19.86-\mathrm{keV}$ resonance, this quantity reduces to $n g \Gamma_{\gamma}$, and because the statistical spin factor is known from previous measurements, the effective areal density $n$ can be derived via RSA from the capture data obtained with the oxide sample. This procedure is valid only if the resonance parameters, and in particular the reaction width, is kept fixed in the fit. In this way, the effective areal density turned out to be $(8.6 \pm 1.0) \times 10^{-3}$ atoms $/ \mathrm{b}$, where the $12 \%$ uncertainty is given by the uncertainty on $\Gamma_{\gamma}$.

This adjusted areal density was used in the RSA of the enriched ${ }^{25} \mathrm{Mg}$ sample. As shown in Fig. 7, the capture yield of the oxide sample can be reproduced with the same accuracy either by fitting the reaction width or the areal density of the sample. On the contrary, the calculation of the yield

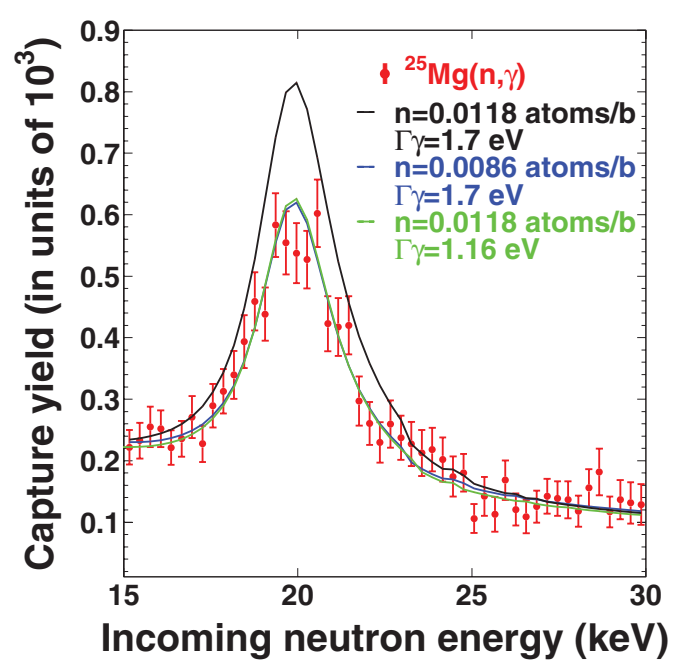

FIG. 7. (Color online) The capture yield of the ${ }^{25} \mathrm{Mg}(n, \gamma)$ resonance at $19.86 \mathrm{keV}$. The data measured with the oxide sample (symbols with error bars) are clearly overestimated with the resonance parameters obtained from the metallic sample and the originally declared areal density (black line). The resonance analysis with SAMMY shows that the data are reproduced treating the areal density $n$ or the capture width $\Gamma_{\gamma}$ as free parameters (blue and green lines, respectively). Consistency with the result obtained with the metal sample requires adopting the reduced areal sample density.

assuming $n=0.01182$ atoms $/ \mathrm{b}$ (i.e., the originally declared value) and $\Gamma_{\gamma}=1.7 \mathrm{eV}$ (derived from the metallic sample) does not reproduce the data. It is obvious that the results for the 19.86-keV resonance can be reconciled only if the reduced areal density is adopted for the enriched sample. The corresponding results of the RSA are listed in Table III together with the uncertainties from the SAMMY fits. Examples of the RSA analysis are given in Figs. 4 and 8.

The parameters of the $19.86-\mathrm{keV}$ resonance derived from the metallic ${ }^{\text {nat }} \mathrm{Mg}$ sample are in agreement with those of Ref. [9]. Moreover, the spin and parity assignment of this level was confirmed since the $\chi^{2} / \mathrm{DOF}\left(\chi^{2}\right.$ is chi-squared and DOF is the number of degrees of freedom) value of the fit (in the energy range $15-30 \mathrm{keV}$ ) was 1.6 assuming $J^{\pi}=2^{+}$and 5.6 assuming $J^{\pi}=3^{+}$. The spin and parity of the $72.66 \mathrm{keV}$ was confirmed as well: the $\chi^{2} / \mathrm{DOF}$ value of the fit (in the energy range 60-80 keV) was 4.6 assuming $J^{\pi}=2^{+}$and 7.9 assuming $J^{\pi}=3^{+}$. The parity of the $62.727-\mathrm{keV}$ resonance was changed to negative according to a recent photoexcitation experiment by Longland et al. [40]. The doubtful resonances at $\approx 102$ and $107 \mathrm{keV}$ were found to improve the quality of the simultaneous RSA and are, therefore, included in Table III. However, they affect the resonance parameters of the large $s$ wave at $100.03 \mathrm{keV}$ and capture data with an enriched sample and very high statistic are required to solve this point. The spin and parity of the $211.14-\mathrm{keV}$ resonance was changed from $3^{-}$ [8] to $2^{-}$, because the simultaneous RSA of the transmission and capture data were more satisfactory. In particular, the $\chi^{2} /$ DOF value of the fit (in the energy range $200-220 \mathrm{keV}$ ) was 1.1 assuming $J^{\pi}=2^{-}$and 3.5 assuming $J^{\pi}=3^{-}$. For the resonances above $450 \mathrm{keV}$ the large statistical uncertainties prevented a meaningful RSA of the capture data. 
TABLE III. ${ }^{25} \mathrm{Mg}+n$ resonance parameters extracted from the simultaneous $R$-matrix analysis, uncertainties include the one from the sample mass. Spin and parity from Ref. [8]. Resonances and values in brackets must be considered with some caution.

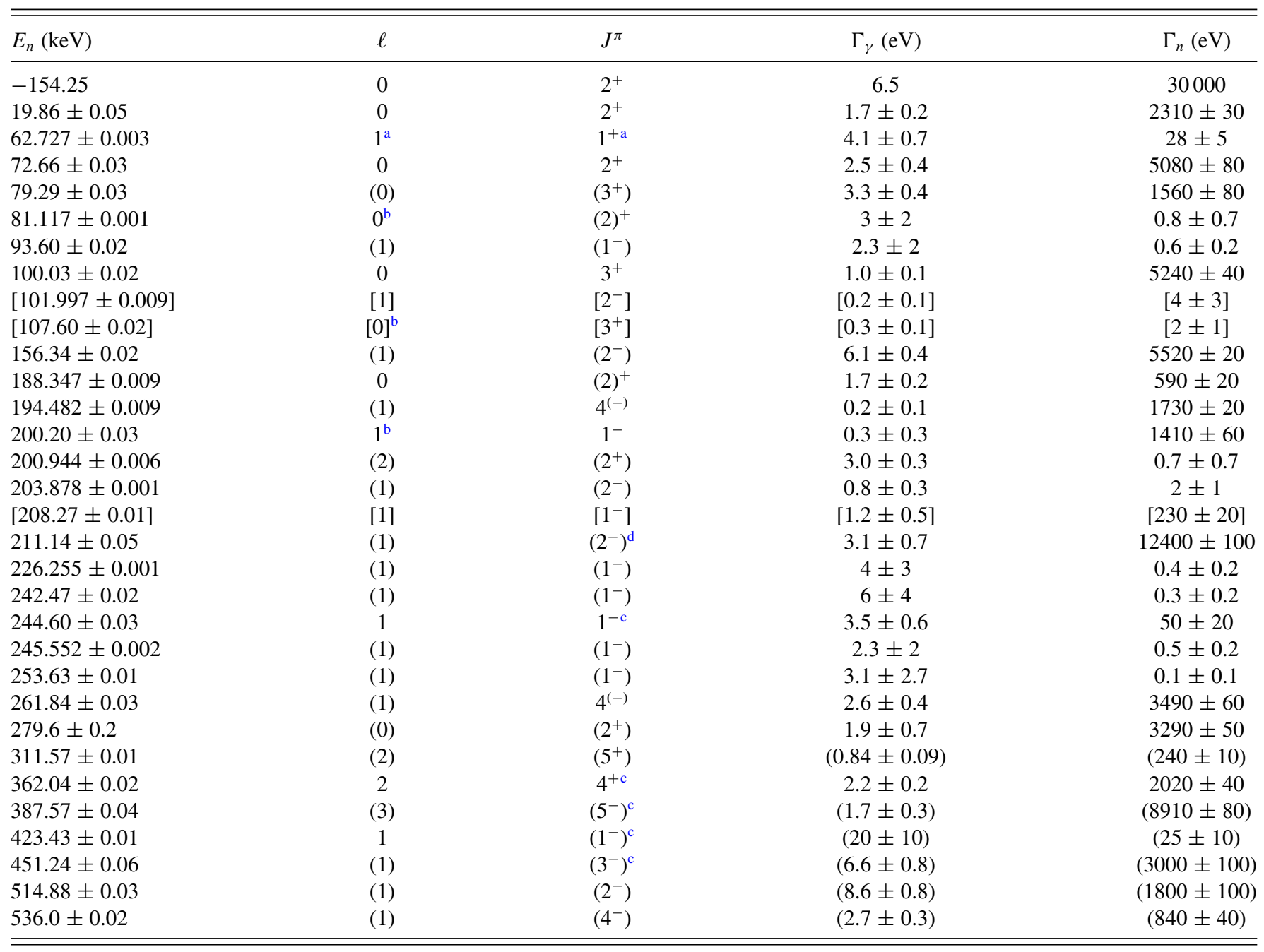

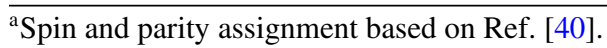

${ }^{\text {b}}$ Parity assignment based on Ref. [19].

${ }^{\mathrm{c}}$ Observed in $\left.{ }^{22} \mathrm{Ne}(\alpha, n)\right)^{25} \mathrm{Mg}$ reaction.

${ }^{\mathrm{d}}$ Spin assignment based on $\chi^{2}$.

\section{C. ${ }^{26} \mathrm{Mg}+n$ resonances}

This analysis is based on the capture yield measured with the enriched ${ }^{26} \mathrm{MgO}$ sample. The RSA results are reported in Table IV together with the uncertainties from the fitting procedure. As indicated by the capture yields in Fig. 2, the mass of the ${ }^{26} \mathrm{Mg}$ sample seemed less affected by adsorption of moisture. Nevertheless, this possibility still implies a significant uncertainty, because none of the resonances could be seen with the natural $\mathrm{Mg}$ sample. Therefore, the $\Gamma_{\gamma}$ values (and, correspondingly, the capture cross section) may be underestimated by as much as $30 \%$.

It is worth noting that capture kernels have been estimated in Ref. [21] on the basis of activation data and a theoretical calculation for the DRC component. The $\omega \gamma$ values for the 68.5- and 219.4-keV resonances are given as $0.067 \pm 0.016 \mathrm{eV}$ and $1.34 \pm 0.24 \mathrm{eV}$, respectively. In the present work we measured $\omega \gamma=0.09 \pm 0.02 \mathrm{eV}$ for the $68.5-\mathrm{keV}$ resonance and $2.17 \pm 0.12 \mathrm{eV}$ for the $219.2-\mathrm{keV}$ resonance. This disagreement would even be enhanced by a possible reduction of the sample mass. Examples of the simultaneous RSA analysis are given in Fig. 9.

TABLE IV. ${ }^{26} \mathrm{Mg}+n$ resonance parameters extracted from the simultaneous $R$-matrix analysis. The quoted uncertainties were obtained by the SAMMY fit. Spin and parity from Ref. [8].

\begin{tabular}{lcccc}
\hline \hline$E_{n}(\mathrm{keV})$ & $\ell$ & $J^{\pi}$ & $\Gamma_{\gamma}(\mathrm{eV})$ & $\Gamma_{n}(\mathrm{eV})$ \\
\hline-60 & 0 & $0.5^{+}$ & 3 & 2400 \\
$68.529 \pm 0.001$ & $(1)$ & $\left(0.5^{-}\right)$ & $0.09^{\mathrm{a}} \pm 0.02$ & $48 \pm 2$ \\
$219.395 \pm 0.002$ & 2 & $1.5^{+}$ & $1.10^{\mathrm{a}} \pm 0.06$ & $80 \pm 2$ \\
$302.34 \pm 0.1 \pm 0.08$ & 1 & $0.5^{-}$ & $6.3^{\mathrm{a}} \pm 0.9$ & $61200 \pm 200$ \\
$427.23 \pm 0.02$ & $(0)$ & $\left(0.5^{+}\right)$ & $2.7^{\mathrm{a}} \pm 0.5$ & $90 \pm 20$ \\
$438.59 \pm 0.09$ & $(1)$ & $\left(0.5^{-}\right)$ & $3.4^{\mathrm{a}} \pm 0.9$ & $11900 \pm 200$ \\
$500.50 \pm 0.01$ & $(1)$ & $\left(1.5^{-}\right)$ & $0.48^{\mathrm{a}} \pm 0.08$ & $260 \pm 10$ \\
\hline \hline
\end{tabular}

${ }^{\mathrm{a}}$ Values may be underestimated by as much as $30 \%$. 
TABLE V. Maxwellian-averaged capture cross sections of ${ }^{24,25,26} \mathrm{Mg}$ (in $\mathrm{mb}$ ) for different temperatures compared with the values in the KADoNiS database [41].

\begin{tabular}{|c|c|c|c|c|c|}
\hline \multirow{2}{*}{$\begin{array}{l}\text { Thermal energy } \\
(\mathrm{keV})\end{array}$} & \multicolumn{3}{|c|}{ This work } & \multicolumn{2}{|c|}{ KADoNiS [41] } \\
\hline & Resonances & DRC & Total & Resonances & Total \\
\hline \multicolumn{6}{|c|}{${ }^{24} \mathrm{Mg}(n, \gamma){ }^{25} \mathrm{Mg}$} \\
\hline 5 & $0.17 \pm 0.01$ & 0.04 & $0.21 \pm 0.01$ & 0.11 & \\
\hline 8 & $0.38 \pm 0.02$ & 0.05 & $0.43 \pm 0.02$ & & \\
\hline 10 & $0.67 \pm 0.04$ & 0.06 & $0.71 \pm 0.04$ & 0.48 & \\
\hline 15 & $1.7 \pm 0.1$ & 0.08 & $1.71 \pm 0.1$ & 1.3 & \\
\hline 20 & $2.7 \pm 0.1$ & 0.09 & $2.7 \pm 0.1$ & 2.3 & \\
\hline 23 & $3.1 \pm 0.2$ & 0.1 & $3.2 \pm 0.2$ & & \\
\hline 25 & $3.3 \pm 0.2$ & 0.1 & $3.4 \pm 0.2$ & 2.9 & \\
\hline 30 & $3.7 \pm 0.2$ & 0.1 & $3.8 \pm 0.2$ & $3.3 \pm 0.4$ & \\
\hline 40 & $3.8 \pm 0.2$ & 0.1 & $3.9 \pm 0.2$ & 3.6 & \\
\hline 50 & $3.6 \pm 0.2$ & 0.1 & $3.7 \pm 0.2$ & 3.4 & \\
\hline 60 & $3.2 \pm 0.2$ & 0.2 & $3.4 \pm 0.2$ & 3.1 & \\
\hline 80 & $2.6 \pm 0.2$ & 0.2 & $2.8 \pm 0.2$ & 2.7 & \\
\hline 90 & $2.4 \pm 0.3$ & 0.2 & $2.6 \pm 0.3$ & & \\
\hline 100 & $2.1 \pm 0.2$ & 0.2 & $2.3 \pm 0.3$ & 2.1 & \\
\hline \multicolumn{6}{|c|}{${ }^{25} \mathrm{Mg}(n, \gamma)^{26} \mathrm{Mg}$} \\
\hline 5 & $3.5 \pm 0.4$ & 0.02 & $3.5 \pm 0.4$ & 4.8 & \\
\hline 8 & $4.9 \pm 0.6$ & 0.03 & $4.9 \pm 0.6$ & & \\
\hline 10 & $5.1 \pm 0.6$ & 0.03 & $5.1 \pm 0.6$ & 5.0 & \\
\hline 15 & $4.9 \pm 0.6$ & 0.03 & $4.9 \pm 0.6$ & 5.5 & \\
\hline 20 & $4.6 \pm 0.4$ & 0.04 & $4.6 \pm 0.4$ & 6.0 & \\
\hline 23 & $4.5 \pm 0.6$ & 0.05 & $4.6 \pm 0.6$ & & \\
\hline 25 & $4.3 \pm 0.6$ & 0.05 & $4.4 \pm 0.6$ & 6.2 & \\
\hline 30 & $4.0 \pm 0.6$ & 0.05 & $4.1 \pm 0.6$ & $6.4 \pm 0.4$ & \\
\hline 40 & $3.4 \pm 0.6$ & 0.06 & $3.5 \pm 0.6$ & 6.2 & \\
\hline 50 & $2.8 \pm 0.5$ & 0.07 & $2.9 \pm 0.5$ & 5.7 & \\
\hline 60 & $2.4 \pm 0.4$ & 0.08 & $2.5 \pm 0.4$ & 5.3 & \\
\hline 80 & $1.8 \pm 0.3$ & 0.09 & $1.9 \pm 0.3$ & 4.4 & \\
\hline 90 & $1.5 \pm 0.2$ & 0.1 & $1.6 \pm 0.2$ & & \\
\hline 100 & $1.3 \pm 0.2$ & 0.1 & $1.4 \pm 0.2$ & 3.6 & \\
\hline \multicolumn{6}{|c|}{${ }^{26} \mathrm{Mg}(n, \gamma)^{27} \mathrm{Mg}$} \\
\hline 5 & $0.067^{\mathrm{a}} \pm 0.002$ & 0.02 & $0.087^{b} \pm 0.002$ & & 0.103 \\
\hline 8 & $0.050^{\mathrm{a}} \pm 0.001$ & 0.02 & $0.070^{\mathrm{b}} \pm 0.001$ & & \\
\hline 10 & $0.047^{\mathrm{a}} \pm 0.001$ & 0.03 & $0.077^{b} \pm 0.001$ & & 0.091 \\
\hline 15 & $0.056^{\mathrm{a}} \pm 0.003$ & 0.03 & $0.086^{b} \pm 0.003$ & & 0.098 \\
\hline 20 & $0.069^{\mathrm{a}} \pm 0.005$ & 0.04 & $0.109^{\mathrm{b}} \pm 0.005$ & & 0.110 \\
\hline 23 & $0.07^{\mathrm{a}} \pm 0.01$ & 0.04 & $0.11^{\mathrm{b}} \pm 0.01$ & & \\
\hline 25 & $0.08^{\mathrm{a}} \pm 0.01$ & 0.04 & $0.12^{b} \pm 0.01$ & & $0.124 \pm 0.008$ \\
\hline 30 & $0.09^{\mathrm{a}} \pm 0.01$ & 0.05 & $0.14^{\mathrm{b}} \pm 0.01$ & $0.084 \pm 0.005$ & $0.126 \pm 0.009$ \\
\hline 40 & $0.12^{\mathrm{a}} \pm 0.01$ & 0.06 & $0.18^{b} \pm 0.01$ & & 0.143 \\
\hline 50 & $0.17^{\mathrm{a}} \pm 0.02$ & 0.06 & $0.23^{b} \pm 0.02$ & & 0.161 \\
\hline 60 & $0.21^{\mathrm{a}} \pm 0.02$ & 0.07 & $0.28^{b} \pm 0.02$ & & 0.165 \\
\hline 80 & $0.29^{\mathrm{a}} \pm 0.04$ & 0.08 & $0.37^{\mathrm{b}} \pm 0.04$ & & 0.226 \\
\hline 90 & $0.31^{\mathrm{a}} \pm 0.05$ & 0.09 & $0.40^{\mathrm{b}} \pm 0.05$ & & \\
\hline 100 & $0.34^{\mathrm{a}} \pm 0.05$ & 0.09 & $0.43^{b} \pm 0.05$ & & 0.265 \\
\hline
\end{tabular}

${ }^{\mathrm{a}}$ Values may be underestimated by as much as $30 \%$.

${ }^{b}$ Values may be underestimated by as much as $20 \%$.

\section{STELLAR CROSS SECTIONS}

\section{A. Resonance contributions}

The cross sections determined using the resonance parameters in Secs. IV A, IV B, and IV C have been convoluted with a Maxwellian neutron energy distribution to obtain the actual stellar cross section (MACS). The results are listed in the Table V for thermal energies between $k T=5$ and $100 \mathrm{keV}$, including the specific values for the common $s$-process sites, e.g., for $k T=8$ and $23 \mathrm{keV}$ related to $\mathrm{He}$ shell burning in low-mass AGB stars and $k T=25$ and $90 \mathrm{keV}$ for the case of core $\mathrm{He}$ and shell $\mathrm{C}$ burning in massive stars. The value 


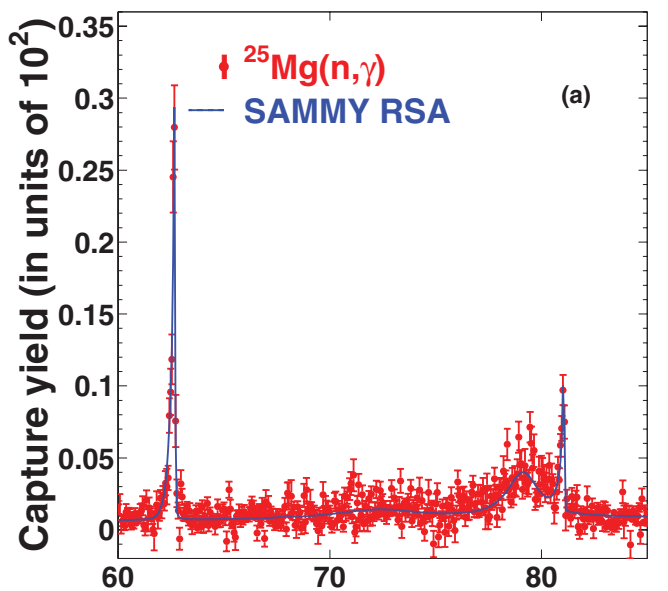

Incoming neutron energy (keV)

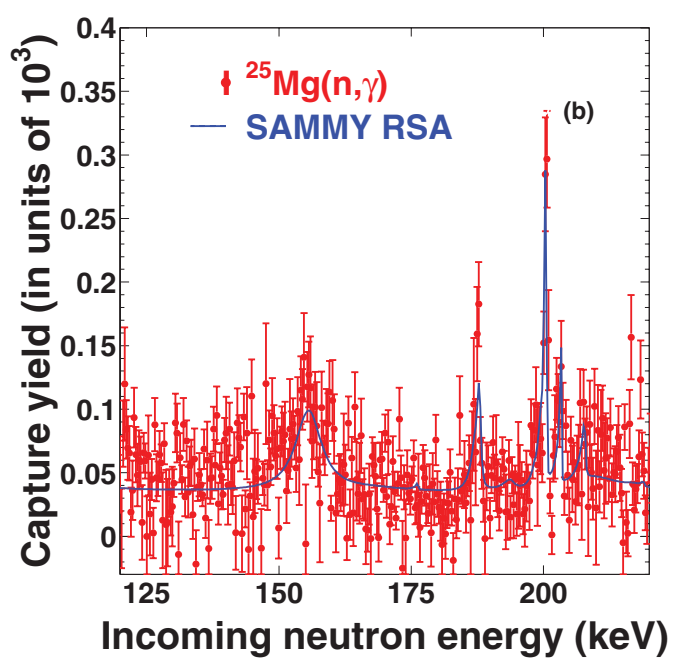

FIG. 8. (Color online) Fits of the capture yield of the ${ }^{25} \mathrm{Mg}+n$ reaction in different energy regions.

for $k T=30 \mathrm{keV}$ is traditionally used for comparison with previous work.

In the resonance analysis, the DRC component is not considered and, therefore, this component has been calculated, as explained in the next section, Sec. V B. The DRC component in the capture process varies smoothly with neutron energy. In a TOF measurement, it cannot be easily disentangled from the background, unless it is much larger than the background level itself. This is not the case in the present experiment, therefore, DRC was considered as a background and was subtracted from the capture yield and from the resonance cross section as well. In order to obtain the actual MACS we added the calculated DRC cross section to those extracted by the resonance shape analysis.

\section{B. Direct capture contributions}

An estimate of the DRC component can be provided by model calculations. In the present work, we adopted the method described in Ref. [42] based on Lane-Lynn theory, developed in the 1960s. Bound-state and scattering-state wave

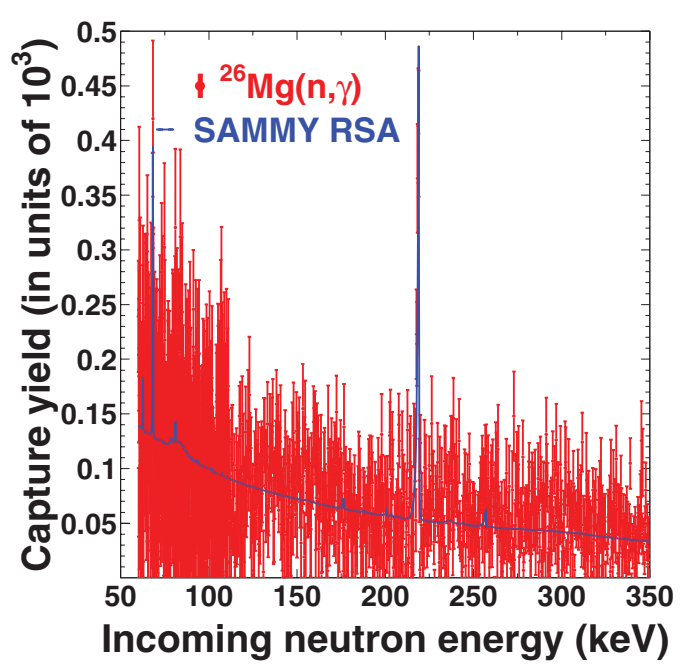

FIG. 9. (Color online) Fits of the capture yield of the ${ }^{26} \mathrm{Mg}+n$ reaction.

functions are derived from a mean-field interaction potential of Woods-Saxon shape with the following common geometric parameters: radius $r_{0}=1.236 \mathrm{fm}$, diffuseness $d=0.62 \mathrm{fm}$, and spin-orbit strength $V_{\mathrm{so}}=7.0 \mathrm{MeV}$. The potential strength for the bound states (final states in the capture process) were fixed assuming the experimental binding energies of the relevant low-lying states. For ${ }^{25} \mathrm{Mg}$ and ${ }^{26} \mathrm{Mg}$, the spectroscopic strengths were assumed to be unity for the states with dominant $1 d_{5 / 2}, 2 s_{1 / 2}$, and $1 d_{3 / 2}$ single-particle character, whereas, for ${ }^{27} \mathrm{Mg}$, experimental values available from the neutron transfer $(d, p)$ reaction were used.

For the calculation of the scattering-state wave functions, two cases have been considered: a plane-wave approximation and a mean interaction potential, whose strength had to be adjusted using some additional assumption. For the latter case, one could consider the same potential strength that binds the ground state of the composite system. Alternatively, a way to derive the mean-field interaction potential could be that of reproducing the neutron-scattering length which, however, would be accurate for incident $s$-wave neutrons, whereas in the present case the dominant electromagnetic transition strength for dipole transitions are for incident $p$-wave neutrons. Given this indetermination, we will provide the result of the MACS for a very wide range of interaction potentials, namely for strengths between $V_{0}=40 \mathrm{MeV}$ and $V_{0}=50 \mathrm{MeV}$.

The results of the calculations for the MACS at $k T=$ $25 \mathrm{keV}$ are shown in Table VI. As can be seen, the resulting DRC cross sections depend strongly on the interaction potential used to derive the scattering-state wave functions. This is due to the fact that for these nuclei, the single-particle $2 p_{3 / 2}$ and $2 p_{1 / 2}$ orbits are located close to the neutron separation energy of the composite systems. The matrix elements are, therefore, in this particular case, very sensitive to the interaction potential strength. As mentioned above, the interaction strength can be constrained further using the information of the neutronscattering length. For ${ }^{24} \mathrm{Mg}$ and ${ }^{26} \mathrm{Mg}$ this leads to interaction potential strengths of $V_{0} \approx 46 \mathrm{MeV}$ and $V_{0} \approx 48 \mathrm{MeV}$, respectively. With these two limiting cases for the potential 
TABLE VI. DRC components of the cross sections. MACS values in $\mu \mathrm{b}$ at $k T=25 \mathrm{keV}$ are given for the different assumptions on the strength of the interaction potential used in the calculation of the scattering wave function. For the Woods-Saxon potential, the two limits correspond to interaction strengths of $V_{0}=40$ and $50 \mathrm{MeV}$. The recommended values are derived as described in the text.

\begin{tabular}{lccc}
\hline \hline Target & Plane wave & Woods-Saxon & Recommended \\
\hline${ }^{24} \mathrm{Mg}$ & 68.4 & $48.6-172.4$ & $102 \pm 15$ \\
${ }^{25} \mathrm{Mg}$ & 25.1 & $21.4-91.7$ & $48 \pm 8$ \\
${ }^{26} \mathrm{Mg}$ & 34.7 & $33.1-180.6$ & 44 \\
\hline \hline
\end{tabular}

strength we have derived the recommended values shown in the fourth column of Table VI.

For ${ }^{24} \mathrm{Mg}$ and ${ }^{25} \mathrm{Mg}$, the DRC component of the cross section remains a small fraction of the resonant component derived from the present analysis, even assuming the largest value resulting from the strongest scattering potential.

For ${ }^{26} \mathrm{Mg}$, the DRC component can be estimated from the difference between the MACS measured via activation $(0.124 \pm 0.008 \mathrm{mb}$ [20], which includes both DRC and resonance components) and our resonance results in Table $\mathrm{V}$. The result $(0.08 \pm 0.01 \mathrm{mb})$ indicates that the DRC is about $40 \%$ of the total capture cross section for this nuclide. The wide range of the results of the DRC model calculation shown in Table VI is compatible with this estimate.

\section{Discussion of uncertainties}

In Table II, Table III, Table IV, and Table V the results of the present experiment are presented together with their uncertainties. These uncertainties are the sum of uncorrelated or statistical uncertainties and systematic uncertainties.

The first component, attributable to counting statistic was obtained from the resonance shape analysis. In particular in the sequential RSA of capture and transmission data, the covariance matrix was used to propagate uncertainties on resonance parameters.

The correlated uncertainties consisted of several components. They come from the PHWT, from the background determination, and from the absolute neutron flux determination. The uncertainty related to the influence of the weighting function was important in this case, since the capture data were normalized to another isotope. The $\gamma$-ray spectra differ markedly because of the large difference in the number of levels available for decay after capture. Therefore, the influence of the detector threshold may play a role. The comparison of partial widths extracted independently from transmission and from capture data gave coherent results within few percentages. From these considerations, we could assign an uncertainty of $3 \%$ from the application of the PHWT. The background determined by the measurement without sample was normalized by means of the number of protons per pulse, which carries an uncertainty of $2 \%$. For the estimation of the uncertainty of the shape of the neutron flux, we adopted an uncertainty of $2 \%$. An uncertainty in the absolute level of the flux is not relevant since we normalized the yield on the saturated resonances. The uncertainty related to the alignment of the sample was estimated to be less than $1 \%$. The combination of these components resulted in a total correlated uncertainty of $4 \%$.

An additional component arises for the powder samples. In the case of ${ }^{25} \mathrm{Mg}$ it was possible to quantify this uncertainty to be $12 \%$, yielding a total systematic uncertainty of $13 \%$. In the case of ${ }^{26} \mathrm{Mg}$ we can argue only that the present result may be underestimated by as much as $30 \%$, which is the magnitude of the correction for ${ }^{25} \mathrm{Mg}$.

The values of the total uncertainty were obtained using SAMMY. The systematic uncertainty was included by propagating the uncertainty of few parameters: the background, the normalization, and, only for the ${ }^{25} \mathrm{Mg}$ sample, the areal density. The uncertainty on background parameter was $20 \%$ and its effect was found to be negligible. The normalization parameter grouped together with the remaining sistematyc uncertaities; therefore, its uncertainty was $4 \%$. In the analysis of ${ }^{25} \mathrm{Mg}$ the areal density was allowed to vary within $12 \%$. Its effect was verified by repeating the RSA of ${ }^{25} \mathrm{Mg}$ with a lower (higher) value of the areal density by $12 \%$. Coherent results were obtained.

In order to propagate the uncertainties from resonances parameters to the stellar cross section, a parametric procedure was adopted. In this procedure, the MACS (in Table V) were calculated using resonance parameters randomly varied within uncertainties.

\section{ASTROPHYSICAL IMPLICATIONS}

\section{A. Impact on $s$-process abundances}

The inventory of the $s$-process abundances in the solar system is provided by the contribution of different type of stars. The so-called main component originates from low-mass asymptotic giant branch (AGB) stars in the range of 1 to 3 solar masses $\left(M_{\odot}\right)$ and is responsible for the $s$ component of the elements between $\mathrm{Sr}$ and the $\mathrm{Pb} / \mathrm{Bi}$ region. The complementary weak component is provided by massive stars with $M \geqslant 8 M_{\odot}$ and is responsible for the production of most of the $s$-process species between Fe and $\mathrm{Sr}$.

Neutron production in low-mass stars occurs during the $\mathrm{He}$ shell burning phase of evolution by recurrent $\mathrm{H}$ and $\mathrm{He}$ burning episodes. By far most of the neutrons are produced by $(\alpha, n)$ reactions on ${ }^{13} \mathrm{C}$ during the quiescent $\mathrm{H}$ burning stage and only about $5 \%$ of the neutron balance is contributed by ${ }^{22} \mathrm{Ne}(\alpha, n){ }^{25} \mathrm{Mg}$ reactions at the higher temperatures reached in the relatively short $\mathrm{He}$ flashes. Accordingly, the production of $\mathrm{Mg}$ is limited so the neutron poisons affecting the main component are dominated by the lighter elements with $Z \leqslant 20$.

The consequences for the abundances produced by the main $s$ component has been studied for the cases describing the solution for the solar $s$ component [43]. In these calculations, only marginal differences of less than $1 \%$ were found if the MACS values of the KADoNiS compilation [41] were replaced by the present results. The main reasons for this negligible impact are (i) that the MACS of ${ }^{25} \mathrm{Mg}$ at the thermal energy of $k T=8 \mathrm{keV}$, which is characteristic of the dominant neutron source provided by the ${ }^{13} \mathrm{C}(\alpha, n){ }^{16} \mathrm{O}$ 
reaction, is nearly identical to the KADoNiS value and (ii) that the ${ }^{22} \mathrm{Ne}(\alpha, n){ }^{25} \mathrm{Mg}$ reaction is only marginally activated in low-mass AGB stars with nearly solar metallicity.

In contrast to low-mass AGB stars, neutron production in massive stars is dominated by the ${ }^{22} \mathrm{Ne}(\alpha, n)^{25} \mathrm{Mg}$ reaction during the convective core He burning and convective shell $\mathrm{C}$ burning phases. Carbon mainly burns via the reaction channels ${ }^{12} \mathrm{C}\left({ }^{12} \mathrm{C}, \alpha\right){ }^{20} \mathrm{Ne}$ and ${ }^{12} \mathrm{C}\left({ }^{12} \mathrm{C}, p\right){ }^{23} \mathrm{Na}$, thus providing the $\alpha$ particles for ${ }^{22} \mathrm{Ne}(\alpha, n)^{25} \mathrm{Mg}$ reactions on the ${ }^{22} \mathrm{Ne}$, left behind at previous core $\mathrm{He}$ exhaustion.

Convective $\mathrm{C}$ shell burning is characterized by a short time scale of the order of 1 year, and a by high neutron density of up to $10^{12} \mathrm{~cm}^{-3}$. The $s$-process nucleosynthesis during this phase was first studied in detail 20 years ago [44] and was confirmed by full stellar evolution calculations up to the supernova explosion [45-47]. In a typical $25 M_{\odot}$ star, the convective $\mathrm{C}$ shell burning zone extends from about 2 to $6 M_{\odot}$, close to the maximum extension of the previous convective $\mathrm{He}$ burning core. The abundances from the inner zone below about $3.5 M_{\odot}$ that are ejected are modified in the final supernova explosion, which essentially destroys the previously produced $s$-process abundances by photodisintegration. The major part of the $s$-process material in the outer zones of the $\mathrm{C}$ burning shell is ejected almost unchanged, forming most of the $s$ process yields of a $25 M_{\odot}$ star [48-50].

Because of the dominance of the ${ }^{22} \mathrm{Ne}(\alpha, n)^{25} \mathrm{Mg}$ reaction as a neutron source in massive stars, a ${ }^{25} \mathrm{Mg}$ nucleus is produced along with practically each free neutron. Therefore, because of its relatively high MACS, ${ }^{25} \mathrm{Mg}$ is a relevant neutron poison. In this section, we study the impact of the present $\mathrm{Mg}$ MACS on full stellar model calculations for a $25 M_{\odot}$ star, with solar metallicity, which were performed with an updated postprocessing code described in Ref. [51].

The effect of the $\mathrm{Mg}$ cross sections from this work is illustrated in Fig. 10. Figure 10(a) shows the $s$-process abundance distributions calculated with the set of MACS values from the KADoNiS database [41] (blue squares) and after the MACS of the Mg isotopes were replaced by the present results (red circles). The relative differences of the two distributions are emphasized by their ratio in Fig. 10(b). In the mass region of the weak $s$ process between $A \approx 60$ and 90 one finds a significant enhancement of the abundance distribution, indicating a reduced poisoning effect. This reduction is mainly due to the lower MACS of ${ }^{25} \mathrm{Mg}$.

Neutron-rich species following the branching points along the $s$-process path $\left({ }^{64} \mathrm{Ni},{ }^{70} \mathrm{Zn},{ }^{76} \mathrm{Ge},{ }^{82} \mathrm{Se},{ }^{86} \mathrm{Kr},{ }^{96} \mathrm{Zr}\right.$, and ${ }^{100}$ Mo in Fig. 10) show a strongest enhancement compared to other isotopes in the same mass region. Some of them, like ${ }^{76} \mathrm{Ge}$ and ${ }^{100} \mathrm{Mo}$, are considered to be mostly produced in $r$-process conditions. The reason of this specific effect is that the reduced poisoning effect results not only in an enhanced efficiency propagated over all the $s$-process distribution but also in a higher neutron density, causing a stronger neutron channel at the different branching points. Apart from these cases, the average enhancement of about $30 \%$ underlines the importance of reliable cross-section data for the light isotopes below the mass range of the Fe peak. As demonstrated by the present results for the $\mathrm{Mg}$ isotopes, these elements can strongly influence the neutron balance of the $s$ process with significant
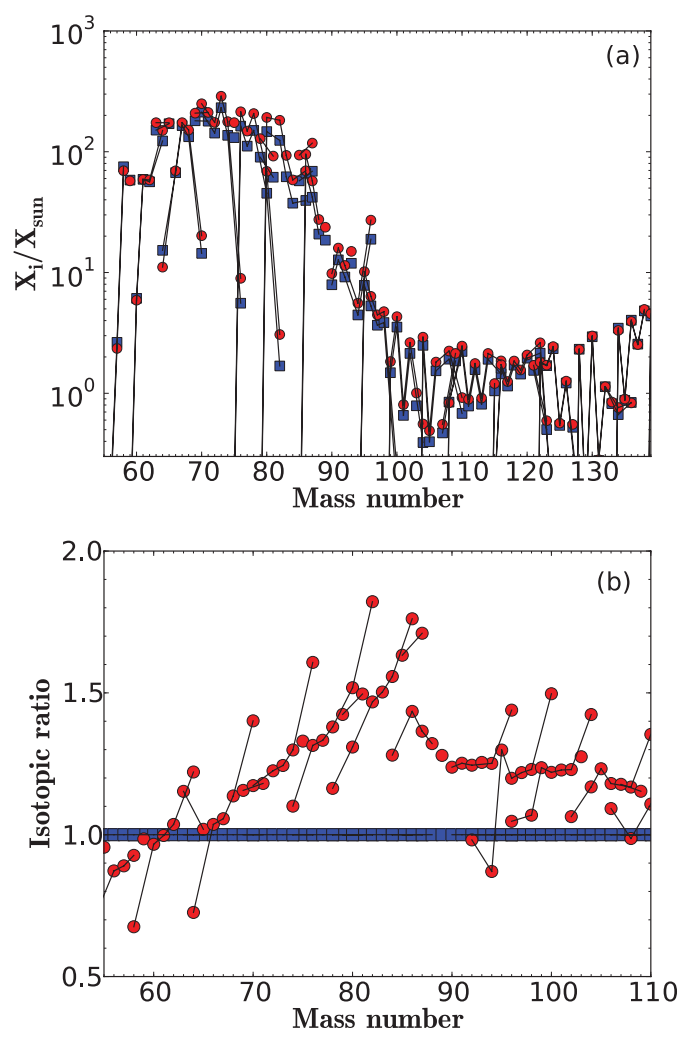

FIG. 10. (Color online) (a) Abundance distribution of the weak $s$ process calculated with MACS values from the KADoNiS database [41] (blue squares) and with the MACS of the Mg isotopes by the present results (red circles). (b) The ratio of the two distributions indicates an average enhancement of $30 \%$ due to the reduced neutron poisoning effect.

consequences for the overall abundance distribution as well as for the analysis of $s$-process branchings.

\section{B. Constraints for the ${ }^{22} \mathrm{Ne}(\alpha, n)^{25} \mathrm{Mg}$ reaction}

As outlined in the previous section, the $(\alpha, n)$ reaction on ${ }^{22} \mathrm{Ne}$ represents an important $s$-process neutron source. Despite several attempts to measure the reaction cross section [52-55], these experiments failed to reach the low $\alpha$ energies of astrophysical relevance, mostly because cosmic-ray induced background starts to dominate the experimental signatures below $E_{\alpha}=0.5 \mathrm{MeV}$. A series of indirect measurements via $\alpha$-particle transfer reactions have been performed to overcome this limitation [56,57]. Alternatively, the ${ }^{25} \mathrm{Mg}(n, \gamma)^{26} \mathrm{Mg}$ reaction can be used to populate the same excited states as shown in Fig. 11.

The reaction rates for the ${ }^{22} \mathrm{Ne}+\alpha$ reaction at $s$-process temperatures are determined by the level structure of the compound nucleus ${ }^{26} \mathrm{Mg}$ above the $\alpha$ threshold $(Q=10.615 \mathrm{MeV})$ and near the neutron threshold $\left(S_{n}=11.093 \mathrm{MeV}\right)$. Previous evaluations [52] have assumed that all states observed by neutron spectroscopy below the lowest observed resonance at $E_{\alpha}=832 \mathrm{keV}$ can contribute to the reaction rate. This approach overestimates this contribution because in the ${ }^{22} \mathrm{Ne}(\alpha, n){ }^{25} \mathrm{Mg}$ reaction the ${ }^{26} \mathrm{Mg}$ compound states are formed 


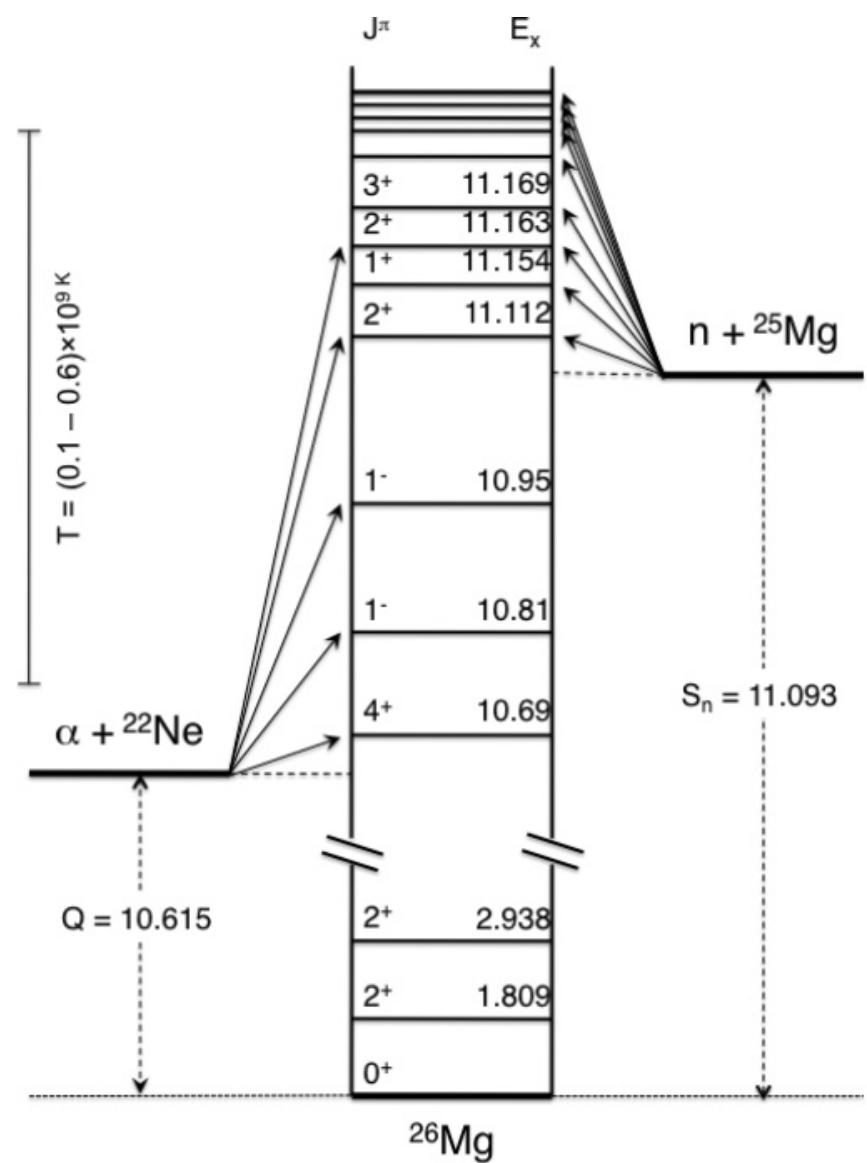

FIG. 11. Level scheme (not to scale) of ${ }^{26} \mathrm{Mg}$ with the ${ }^{22} \mathrm{Ne}+\alpha$ and ${ }^{25} \mathrm{Mg}+n$ entrance channels. The reaction $Q$ values and the neutronseparation energy $S_{n}$ are in MeV. Spin and parity values of resonances are given together with the level energies. Note the negative $Q$ value for the ${ }^{22} \mathrm{Ne}(\alpha, n)^{25} \mathrm{Mg}$ reaction.

through spin 0 particles. Therefore, the resonance states have natural spin and parity $\left(0^{+}, 1^{-}, 2^{+}, \ldots\right)$ and correspond only to a subset of ${ }^{26} \mathrm{Mg}$ states, which are populated in the ${ }^{25} \mathrm{Mg}(n, \gamma)^{26} \mathrm{Mg}$ reaction. In fact, $s$-wave neutron capture populates $2^{+}, 3^{+}$states, whereas $1^{-}, 2^{-}, 3^{-}, 4^{-}$are reached by $p$-wave capture and $0^{+}, 1^{+}, 2^{+}, 3^{+}, 4^{+}, 5^{+}$by $d$-wave capture. Therefore, only a subset of levels was selected from indirect measurements in Ref. [58] but included still some non-natural states. Recent information on the level scheme of ${ }^{26} \mathrm{Mg}$, obtained from nuclear resonance fluorescence [40] experiments, were used by Longland [59] to improve the calculation of the reaction rate.

The present study provides strong evidence for natural parity for the resonances at $19.86 \mathrm{keV}\left(E_{x}=11.112 \mathrm{MeV}\right)$ and $72.66 \mathrm{keV}\left(E_{x}=11.163 \mathrm{MeV}\right)$. Moreover, the present work is compatible with the results of the experiment by Longland [40] which gives strong indications for non-natural parity for the resonances at $62.727 \mathrm{keV}\left(E_{x}=11.154 \mathrm{MeV}\right)$. For other resonances in the energy region of interest $\left(E_{n} \lesssim 250 \mathrm{keV}\right)$, the spin and parity assignments remain uncertain.

The contribution of narrow resonances to the ${ }^{22} \mathrm{Ne}(\alpha, n)^{25} \mathrm{Mg}$ reaction rate (in $\mathrm{cm}^{3} / \mathrm{s} / \mathrm{mole}$ ) can be

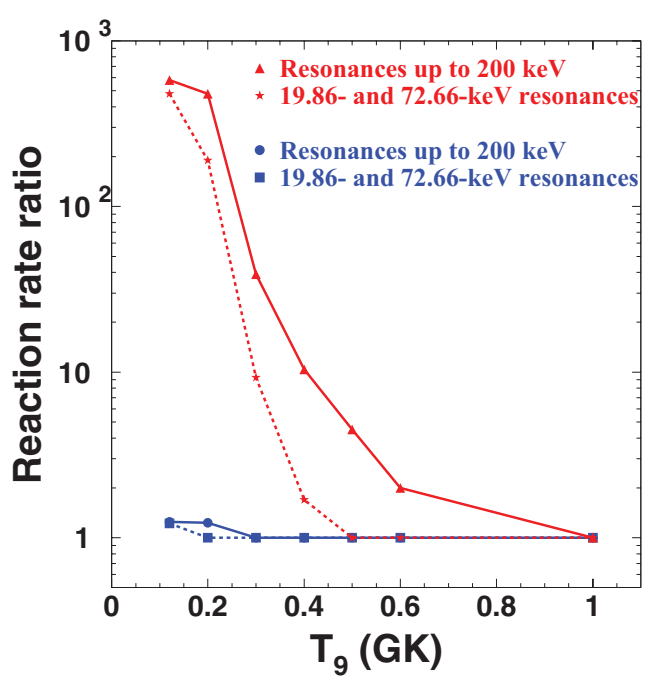

FIG. 12. (Color online) Ratio of the upper and lower limits of the ${ }^{22} \mathrm{Ne}(\alpha, n){ }^{25} \mathrm{Mg}$ reaction rates and of the recommended values from NACRE [61] as a function of temperature. Blue curves are calculated using $\Gamma_{\alpha}$ from Ref. [58], and red curves are calculated using $\Gamma_{\alpha}$ from Ref. [52].

estimated [60] as

$$
N_{A}\langle\sigma v\rangle_{r} \cong \frac{1.54 \times 10^{5}(2 J+1) \Gamma_{\alpha}}{A^{3 / 2} T_{9}^{3 / 2} e^{11.605 E_{R} / T_{9}}}
$$

where $J$ is the spin of the resonance, $\Gamma_{\alpha}$ the $\alpha$ width (in $\mathrm{eV}), A$ the reduced mass, and $T_{9}$ the temperature in GK $\left(T_{9}=T / 10^{9} \mathrm{~K}\right) . E_{R}$ denotes the resonance energy in the center-of-mass system (in MeV). The calculation was repeated assuming different values for $\Gamma_{\alpha}$ (a quantity that cannot be determined by neutron spectroscopy). Lower limits of $\Gamma_{\alpha}$ are from Ref. [58], and upper limits are from Ref. [52]. In Table VII the contributions of the resonances observed in the this work are summarized. In particular, two groups have been considered: (i) the natural-parity resonances at 19.86 and $72.66 \mathrm{keV}$ and (ii) resonances from this work below $244 \mathrm{keV}$ $\left(E_{\alpha}=832 \mathrm{keV}\right)$, where the assignment of natural parity is uncertain $\left(E_{n}=81.117,93.6,188.347,200.2,200.944\right.$, and $208.27 \mathrm{keV})$.

Below $0.2 \mathrm{GK}$ the reaction rate is dominated by the natural parity resonances at 19.86 and $72.66 \mathrm{keV}$. The rate uncertainty is determined by the $\Gamma_{\alpha}$ value but not by the energy [see Eq. (5)], which is precisely inferred from the analysis of the neutron data. However, there are still a few resonances with uncertain spin and parity assignments which could contribute to the ${ }^{22} \mathrm{Ne}(\alpha, n)^{25} \mathrm{Mg}$ reaction rate at and above $0.2 \mathrm{GK}$. Therefore, a combined transmission and capture experiment with highly enriched ${ }^{25} \mathrm{Mg}$ samples would be highly desirable for obtaining unambiguous spins and parities. Moreover, the ${ }^{22} \mathrm{Ne}(\alpha, n)^{25} \mathrm{Mg}$ rate is still suffering from missing experimental information on $\Gamma_{\alpha}$. This is illustrated in Fig. 12 by the comparison of the calculated reaction rate from this work and from the Nuclear Astrophysical Compilation of Reaction rate (NACRE) evaluation [61]. 
TABLE VII. Neutron-resonance contributions (in \%) to the rate of the ${ }^{22} \mathrm{Ne}(\alpha, n)^{25} \mathrm{Mg}$ reaction at different temperatures.

\begin{tabular}{lcccc}
\hline \hline$T_{9}$ & \multicolumn{2}{c}{ Group (i) } & & Group (ii) \\
\cline { 2 - 5 } & $\Gamma_{\alpha}$ from Ref. [58] & $\Gamma_{\alpha}$ from Ref. [52] & $\Gamma_{\alpha}$ from Ref. [58] & 2 \\
$\Gamma_{\alpha}$ from Ref. [52] \\
0.12 & 98 & 83 & 8 & 17 \\
0.2 & 30 & 40 & 3 & 60 \\
0.3 & 2 & 24 & -2 & 74 \\
0.4 & $<1$ & 17 & - & 74 \\
0.5 & - & 12 & - & 72 \\
0.6 & - & 1 & - & 2 \\
\hline \hline
\end{tabular}

\section{CONCLUSIONS}

We have measured the resonance neutron-capture cross section of the three stable isotopes of $\mathrm{Mg}$ from $1 \mathrm{eV}$ to about $700 \mathrm{keV}$ neutron energy. The measurement has been performed with a capture setup optimized for capture cross-section measurement of isotopes showing very large elastic-to-reaction-channel ratios. We have updated the $(n, \gamma)$ cross sections of ${ }^{24,25,26} \mathrm{Mg}$ by combining the present capture data with high-resolution transmission data from ORELA. In this way, the parametrization of the cross section in terms of resonance parameters could be significantly improved. The use of highly enriched samples permitted us to assign doubtful resonances, e.g., the $68.5-\mathrm{keV}$ resonance could be assigned to ${ }^{26} \mathrm{Mg}$ and the $177-\mathrm{keV}$ resonance to ${ }^{24} \mathrm{Mg}$. The spin and parities of the $62.727-\mathrm{keV}$ and $211.14-\mathrm{keV}$ resonances in ${ }^{25} \mathrm{Mg}$ were changed and the evaluation was extended. The present parametrization, including resonances at negative energies, was adapted to reproduce the experimental value of the cross section at thermal energy. Maxwellian average cross sections determined from the present data were found to differ significantly from earlier work. These values were about $20 \%$ higher for ${ }^{24} \mathrm{Mg}$ and about $40 \%$ lower for ${ }^{25} \mathrm{Mg}$ than recommended previously.

The ${ }^{26} \mathrm{Mg}$ results are in agreement with existing data, but with a possible underestimation by $20 \%$, due to sample features. It was shown that the new values reduce the effect of ${ }^{25} \mathrm{Mg}$ as a neutron poison for the $s$ process in massive stars, leading to a higher production propagated over all the $s$-process distribution. In particular, the largest variation is obtained in the $\mathrm{Kr}-\mathrm{Rb}$ region, with an increase up to $50-70 \%$.

Constraints of the present results for the $s$-process neutron source reaction ${ }^{22} \mathrm{Ne}(\alpha, n)^{25} \mathrm{Mg}$ have been discussed, and a new experiment for obtaining definite spin-parity assignments of the important ${ }^{26} \mathrm{Mg}$ levels was proposed.
[1] E. M. Burbidge, G. R. Burbidge, W. A. Fowler, and F. Hoyle, Rev. Mod. Phys 29, 547 (1957).

[2] F. Käppeler, H. Beer, and K. Wisshak, Rep. Prog. Phys. 52, 945 (1989).

[3] R. Gallino, C. Arlandini, M. Busso, M. Lugaro, C. Travaglio, O. Straniero, A. Chieffi, and M. Limongi, Astrophys. J. 497, 388 (1998).

[4] C. Sneden, J. J. Cowan, and R. Gallino, Annu. Rev. Astron. Astrophys. 46, 241 (2008).

[5] C. Iliadis, A. Champagne, A. Chieffi, and M. Limongi, Astrophys. J. Suppl. Ser. 193, 16 (2011).

[6] T. Nakagawa et al., J. Nucl. Sci. Technol. 32, 1259 (1995).

[7] S. F. Mughabghab and D. I. Garber, Neutron Cross Sections (Academic Press, New York, 1984).

[8] P. E. Koehler, Phys. Rev. C 66, 055805 (2002).

[9] H. Weigmann, R. L. Macklin, and J. A. Harvey, Phys. Rev. C 14, 1328 (1976).

[10] K. H. Guber, D. C. Larson, P. E. Koehler, R. R. Spencer, S. Raman, J. A. Harvey, N. W. Hill, T. A. Lewis, and R. R. Winters, in International Conference on Nuclear Data for Science and Technology, edited by G. Reffo, A. Ventura, and C. Grandi (Societa Italiana di Fisica, Bologna, 1997), p. 559.

[11] P. H. Koehler (private communication).

[12] G. Nyström, B. Lundberg, and I. Bergqvist, Phys. Scr. 4, 95 (1971).
[13] D. J. Horen, J. A. Harvey, and N. W. Hill, Phys. Rev. C 15, 1168 (1977)

[14] T. Uchiyama, M. Igashira, and H. Kitazawa, Phys. Rev. C 41, 862 (1990).

[15] H. Kitazawa, M. Igashira, M. Shimizu, K. Muto, T. Oda, Y. Achiha, Y.-H. Lee, and N. Mukai, Phys. Rev. C 46, 2364 (1992)

[16] H. W. Newson, R. C. Block, P. F. Nichols, A. Taylor, A. K. Furr, and E. Merzbacher, Ann. Phys. 8, 211 (1959).

[17] I. Bergqvist, J. A. Biggerstaff, J. H. Gibbons, and W. M. Good, Phys. Rev. 158, 1049 (1967).

[18] U. N. Singh, H. I. Liou, J. Rainwater, G. Hacken, and J. B. Garg, Phys. Rev. C 10, 2150 (1974).

[19] B. L. Berman, R. J. Baglan, and C. D. Bowman, Phys. Rev. Lett. 24, 319 (1970).

[20] P. Mohr, H. Beer, H. Oberhummer, and G. Staudt, Phys. Rev. C 58, 932 (1998).

[21] P. Mohr, H. Beer, H. Oberhummer, W. Rochow, P. V. Sedyshev, S. Volz, and A. Zilges, Phys. Rev. C 60, 017603 (1999).

[22] A. M. Lane and J. E. Lynn, Nucl. Phys. 17, 563 (1960).

[23] T. A. Walkiewicz, S. Raman, E. T. Jurney, J. W. Starner, and J. E. Lynn, Phys. Rev. C 45, 1597 (1992).

[24] F. Gunsing et al. (n_TOF Collaboration), Nucl. Instrum. Methods B 261, 925 (2007).

[25] S. Abramovich et al., Technical Report No. CERN-SPSC-99-8. SPSC-P-310, 1999. 
[26] U. Abbondanno et al., Technical Report No. CERN-SL-2002-053 ECT, 2003.

[27] R. Plag, M. Heil, F. Käppeler, P. Pavlopoulos, R. Reifarth, and K. Wisshak, Nucl. Instrum. Methods A 496, 425 (2003).

[28] A. Borella, G. Aerts, F. Gunsing, M. Moxon, P. Schillebeeckx, and R. Wynants, Nucl. Instrum. Methods A 577, 626 (2007).

[29] S. Marrone et al. (n_TOF Collaboration), Nucl. Instrum. Methods A 517, 389 (2004).

[30] U. Abbondanno et al. (n_TOF Collaboration), Nucl. Instrum. Methods A 538, 692 (2005).

[31] C. Massimi, A. Borella, S. Kopecky, C. Lampoudis, M. C. Moxon, P. Schielleebeckx, and G. Vannini, J. Kor. Phys. Soc. 59, 1689 (2011).

[32] N. A. Melnichenko (private communication).

[33] L. R. Macklin, J. Halperin, and R. R. Winters, Nucl. Instrum. Methods 164, 213 (1979).

[34] U. Abbondanno et al. (n_TOF Collaboration), Nucl. Instrum. Methods A 521, 454 (2004).

[35] H. Weigmann et al.: Data file EXFOR-10791.002, dated 2003-02-11, compare with Phys. Rev. C 14, 1328, (1976), EXFOR data retrieved from the IAEA Nuclear Data Section, Vienna.

[36] N. M. Larson, Updated Users' Guide for SAMMY: Multilevel Rmatrix Fits to Neutron Data Using Bayes' Equations, SAMMY Computer Code, Report No. ORNL/TM-9179/R7, Oak Ridge National Laboratory, 2008.

[37] P. Endt, Nucl. Phys. A 633, 1 (1998).

[38] K. H. Guber, P. E. Koehler, H. Derrien, T. E. Valentine, L. C. Leal, R. O. Sayer, and T. Rauscher, Phys. Rev. C 67, 062802 (2003).

[39] F. H. Fröhner, JEFF Report No. 18, 2000.

[40] R. Longland, C. Iliadis, G. Rusev, A. P. Tonchev, R. J. deBoer, J. Görres, and M. Wiescher, Phys. Rev. C 80, 055803 (2009).

[41] I. Dillmann, R. Plag, F. Käppeler, and T. Rauscher, in EFNUDAT Fast Neutrons: Scientific Workshop on Neutron Measurements, Theory and Applications (JRC-IRMM, Geel, 2009) [http://www.kadonis.org].

[42] A. Mengoni, T. Otsuka, and M. Ishihara, Phys. Rev. C 52, 2334R (1995).

[43] C. Arlandini, F. Käppeler, K. Wisshak, R. Gallino, M. Lugaro, M. Busso, and O. Straniero, Ap. J. 525, 886 (1999).
[44] C. Raiteri, M. Busso, R. Gallino, and G. Picchio, Ap. J. 371, 665 (1991).

[45] M. Limongi, O. Straniero, and A. Chieffi, Ap. J. Suppl. 129, 625 (2000).

[46] S. Woosley, A. Heger, and T. Weaver, Rev. Mod. Phys. 74, 1015 (2002).

[47] L. The, M. El Eid, and B. Meyer, Ap. J. 655, 1058 (2007).

[48] S. E. Woosley and T. A. Weaver, Astrophys. J. Suppl. Ser. 101, 181 (1995).

[49] M. Limongi, O. Straniero, and A. Chieffi, Astrophys. J. Suppl. Ser. 129, 625 (2000).

[50] T. Rauscher, A. Heger, R. D. Hoffman, and S. E. Woosley, Astrophys. J. 576, 323 (2002).

[51] M. Pignatari, R. Gallino, C. Baldovin, M. Wiescher, F. Herwig, A. Heger, M. Heil, and F. Käppeler, in Nuclei in the Cosmos-IX, edited by A. Mengoni et al. (SISSA, Trieste, 2006) [http://pos.sissa.it/, p. contribution 061].

[52] K. Wolke, V. Harms, H. W. Becker, J. W. Hammer, K. L. Kratz, C. Rolfs, U. Schröder, H. P. Trautvetter, M. Wiescher, and A. Wöhr, Z. Phys. A 334, 491 (1989).

[53] V. Harms, K.-L. Kratz, and M. Wiescher, Phys. Rev. C 43, 2849 (1991).

[54] H. W. Drotleff, A. Denker, J. W. Hammer, H. Knee, S. Küchler, D. Streit, C. Rolfs, and H. P. Trautvetter, Z. Phys. A 338, 367 (1991).

[55] M. Jaeger, R. Kunz, A. Mayer, J. W. Hammer, G. Staudt, K. L. Kratz, and B. Pfeiffer, Phys. Rev. Lett. 87, 202501 (2001).

[56] U. Giesen, C. Browne, J. Görres, S. Graff, C. Iliadis, H.-P. Trautvetter, M. Wiescher, W. Harms, K. Kratz, B. Pfeiffer et al., Nucl. Phys. A 561, 95 (1993).

[57] C. Ugalde, A. E. Champagne, S. Daigle, C. Iliadis, R. Longland, J. R. Newton, E. Osenbaugh-Stewart, J. A. Clark, C. Deibel, A. Parikh et al., Phys. Rev. C 76, 025802 (2007).

[58] A. I. Karakas, M. A. Lugaro, M. Wiescher, J. Görres, and C. Ugalde, Astrophys. J. 643, 471 (2006).

[59] R. L. Longland, Ph.D. thesis, University of North Carolina, 2010.

[60] N. A. Bahcall and W. A. Fowler, Astrophys. J. 157, 659 (1969).

[61] C. Angulo, M. Arnould, M. Rayet, P. Descouvemont, D. Baye, C. Leclercq-Willain, A. Coc, S. Barhoumi, P. Aguer, C. Rolfs et al., Nucl. Phys. A 656, 3 (1999). 